\title{
ON THE AUTOMORPHISM GROUP OF A REDUCED PRIMARY ABELIAN GROUP
}

\author{
BY \\ KAI FALTINGS
}

\begin{abstract}
The classical theorems concerning isomorphisms and automorphisms of full linear groups are generalized to reduced primary Abelian groups and their automorphism groups. Also, a duality theory for (not necessarily finite) reduced Abelian p-groups is presented.
\end{abstract}

This paper is an investigation of the relationship between isomorphisms of the automorphism groups and projectivities of the subgroup lattices of reduced primary Abelian groups, generalizing the corresponding results on vector spaces; cf. Baer [2, Chapter VI] and Dieudonné [3, pp. 90-95]. The outline is like in Baer [2], the details, however, are quite different.

In particular, a reduced Abelian $p$-group $A$ may be isomorphic to its adjoint group [ = maximal torsion subgroup of $\operatorname{Hom}\left(A, Z\left(p^{\infty}\right)\right)$ ] without being finite (Lemma 2.2), whereas it is well known that the group $A$ has a dual in the category of Abelian groups if and only if $A$ is finite; cf. Fuchs [4, p. 312].

Our limitations are as follows. Firstly, we require that the Abelian groups under consideration are ( $p p$ )-groups: a reduced Abelian group $A$ is a ( $p p$ )-group if and only if it is a nonzero $p$-group such that, for every cyclic direct summand $P$ of $A$, every complement of $P$ in $A$ has a cyclic direct summand of order greater than or equal to the order of $P$. Secondly, for the same reason as in Leptin's paper [8], we have to require that the prime number involved be greater than 3 .

The organization of the material is as follows. In $\$ 1$ and 2, we collect results of an [more or less] auxiliary nature. In particular, we show that $\operatorname{Hom}(A, B)$ is irreducible as an (Aut $A-$ Aut $B$ )-bimodule if $A$ and $B$ are reduced Abelian $p$-groups with $p \neq 2$ (Lemma 1.9). $\S 2$ contains some results of the duality theory of [not necessarily finite] reduced Abelian $p$-groups, as they are needed in $\$ \$ 4$ and 5.

In $\S 3$, we give a group-theoretical characterisation of stabilizers of direct summands of reduced Abelian p-groups with $p>3$ (Theorem 3.4), and in $\$ 4$ we use this characterisation in order to prove our main theorem (Theorem 4.9).

Presented to the Society, April 5, 1969 under the title Isomorphism of the automorphism groups and projectives of primary abelian groups; received by the editors November 2, 1970.

AMS 1969 subject classifications. Primary 2030.

Key words and phrases. Primary Abelian group, reduced group, automorphism group, subgroup lattice, projectivity, duality, adjoint homomorphism, stabilizer, direct summand, involution, extreme involution. 
Finally, we show that the group of all automorphisms of the automorphism group of a reduced Abelian ( $p p$ )-group with $p>3$ splits, just as in the classical case (Theorem 5.3).

Notation. The notation is standard, with the following possible exceptions:

$[x \mid \ldots]=$ set of all elements $x$ such that...,

$\{\ldots, x, \ldots\}=[$ sub]group generated by the enclosed elements,

$c_{G} H=$ centralizer of $H$ in $G$,

$n_{G} H=$ normalizer of $H$ in $G$,

$c G=c_{G} G=$ center of $G$,

$G \subseteq H:=: G$ is a subgroup of $H$,

$G \subset H:=: G \subseteq H$ and $G \neq H$,

$|G|=$ order of $G$,

$o(x)=$ order of the element $x$,

$\operatorname{Exp} G=\operatorname{exponent}$ of the bounded group $G$,

Aut $G=$ group of all automorphisms of the group $G$.

If $A$ is an Abelian group, then

$n A=[n a \mid a \in A]$ for every integer $n$,

$A[n]=[a \mid a \in A$ and $n a=0]$ for every integer $n$,

End $A=$ ring of all endomorphisms of $A$,

$L A=$ lattice of all subgroups of $A$,

$H A=$ set of all proper direct summands of $A$,

$t A=$ maximal torsion subgroup of $A$,

$\sum_{i} A_{i}=$ subgroup generated by the subgroups $A_{i}$,

$\sum_{i}^{*} A_{i}=$ cartesian sum [= product] of the Abelian groups $A_{i}$,

$\sum_{i}^{\circ} A_{i}=$ direct sum of the Abelian groups $A_{i}$.

If $H$ is a subgroup of the Abelian group $A$, then $\Sigma_{A} H=[\gamma \in$ Aut $A \mid A(\gamma-1) \subseteq H$ and $H(\gamma-1)=0$ ] is the stabilizer of $H$ in $A$.

If $A$ is an Abelian group and if $\alpha$ is an involution [=automorphism of order 2 or 1] of $A$, then

$A_{+}^{\alpha}=[x \mid x \in A$ and $x \alpha=x]$,

$A_{-}^{\alpha}=[x \mid x \in A$ and $x \alpha=-x]$,

$\Sigma_{+}^{\alpha}=\Sigma_{A} A_{+}^{\alpha}$,

$\Sigma_{-}^{\alpha}=\Sigma_{A} A_{-}^{\alpha}$;

$Z\left(p^{\infty}\right)=$ quasicyclic group of type $p^{\infty}$,

$A^{*}=t \operatorname{Hom}\left(A, Z\left(p^{\infty}\right)\right)$ for a reduced Abelian $p$-group $A$.

\section{Preliminaries.}

Definition. Let $A$ be an Abelian group and let $H$ be a subgroup of $A$. Then the stabilizer $\Sigma_{A} H$ of $H$ in $A$ is the group of all automorphisms of $A$ which induce the identity automorphism in both $H$ and $A / H$. We remark that the mapping $\gamma \rightarrow \gamma-1$ induces an isomorphism of $\Sigma_{A} H$ onto $\operatorname{Hom}(A / H, H)$; cf. Hausen [6, Hilfssatz $1.4]$. 
Proposition 1.1. Let $A$ be an Abelian group, let $D$ be a direct summand of $A$ and let $A=D \oplus E=D \oplus F$. Then there exists a unique element $\gamma \in \Sigma_{A} D$ such that $E \gamma=F$.

The straightforward proof is left to the reader.

LEMMA 1.2. Let $A$ be an Abelian p-group. Then the intersection of all complements of cyclic direct summands of $A$ is equal to $p^{\omega} A$.

Proof. By [5, Theorem 9.6, p. 48], the intersection of all complements of a direct summand $B$ of $A$ is equal to the maximal fully invariant subgroup of $A$ disjoint from $B$. Now the maximal fully invariant subgroup of $A$ disjoint from a cyclic direct summand of order $p^{k}$ is $p^{k} A$, and thus the result follows.

The following propositions on involutions of Abelian $p$-groups with $p \neq 2$ are more or less known; the proofs, being straightforward, are left to the reader.

Proposition 1.3. Let $A$ be an Abelian $p$-group with $p \neq 2$ and let $\alpha$ and $\beta$ be involutions of $A$. Then we have

(a) $A=A_{+}^{\alpha} \oplus A_{-}^{\alpha}$,

(b) $c_{\text {Aut } A}\{\alpha\}=\left[\gamma \in\right.$ Aut $A \mid A_{+}^{\alpha} \gamma=A_{+}^{\alpha}$ and $\left.A_{-}^{\alpha} \gamma=A_{-}^{\alpha}\right]$,

(c) $\alpha$ and $\beta$ commute if and only if

$$
A=\left(A_{+}^{\alpha} \cap A_{+}^{\beta}\right) \oplus\left(A_{+}^{\alpha} \cap A_{-}^{\beta}\right) \oplus\left(A_{-}^{\alpha} \cap A_{+}^{\beta}\right) \oplus\left(A_{-}^{\alpha} \cap A_{-}^{\beta}\right) .
$$

Proposition 1.4. Let $A$ be an Abelian $p$-group with $p \neq 2$ and let $\alpha$ and $\beta$ be commuting involutions of $A$. Then the following are equivalent:

(a) $A_{+}^{\alpha} \subseteq A_{-}^{\beta}$,

(b) $A_{+}^{\beta} \subseteq A_{-}^{\alpha}$,

(c) $A_{+}^{\alpha} \cap A_{+}^{\beta}=0$.

Proposition 1.5. Let $A$ be a reduced Abelian p-group with $p \neq 2$ and let $\alpha$ and $\beta$ be commuting involutions of $A$. Then the following are equivalent:

(a) $\Sigma_{+}^{\alpha} \cap \Sigma_{+}^{\beta}=1$,

(b) $\Sigma_{-}^{\alpha} \cap \Sigma_{-}^{\beta}=1$,

(c) $A_{+}^{\alpha} \cap A_{+}^{\beta}=0$ or $A_{-}^{\alpha} \cap A_{-}^{\beta}=0$.

Remark. The proof of Proposition 1.5 is based on property (*) in Proposition 1.3(c) and on the fact that for any two reduced Abelian $p$-groups $A \neq 0$ and $B \neq 0$, there exist homomorphisms $\sigma: A \rightarrow B$ and $\tau: B \rightarrow A$ such that $A \sigma \neq 0$ and $B \tau \neq 0$.

Definition. Let $A$ be a reduced Abelian $p$-group and let $\alpha$ be an involution of $A$. Then $\alpha$ is extreme if and only if the group of all involutions in the center of the centralizer of $\{\alpha, \beta\}$ has order not exceeding 8 for all involutions $\beta$ of $A$ with $\alpha \beta=\beta \alpha$; cf. Dieudonné [3, p. 91].

Proposition 1.6. Let $A$ be a reduced Abelian $p$-group with $p \neq 2$ and let $\alpha$ be an involution of $A$. Then the following are equivalent:

(a) $\alpha$ is extreme,

(b) $A_{+}^{\alpha}$ or $A_{-}^{\alpha}$ is cyclic. 
Remark. The proof of Proposition 1.6 follows from Proposition 1.3(b) and (c) and from the following facts:

(+) A reduced $p$-group is directly indecomposable if and only if it is cyclic; cf. Fuchs $[4$, p. 80].

$(++)$ If $A \neq 0$ is an Abelian $p$-group with $p \neq 2$, then the group of all involutions in the center of Aut $A$ has order 2.

Proposition 1.7. Let $A$ and $B$ be reduced Abelian p-groups and let $\sigma$ be a homomorphism of $A$ into $B$ such that for all homomorphisms $\tau$ of $B$ into $A$ we have $\sigma \tau=0$ and $\tau \sigma=0$. Then $\sigma=0$.

Proof. From $\sigma \tau=0$ and $\tau \sigma=0$ for all $\tau: B \rightarrow A$ we infer

$$
\sum_{\tau: B \rightarrow A} B \tau \subseteq \operatorname{ker} \sigma
$$

and

$$
A \sigma \subseteq \bigcap_{\tau: B \rightarrow A} \operatorname{ker} \tau .
$$

Case 1. $B$ is not bounded or $\operatorname{Exp} B \geqq \operatorname{Exp} A$. Then for all $x \in A$, there exists a direct summand $\{y\}$ of $B$ with $o(y) \geqq o(x)$; and consequently, there exists a homomorphism $\tau: B \rightarrow A$ with $y \tau=x$. Hence $\sum_{\tau: B \rightarrow A} B \tau=A$ and from (1) we get $A$ $=\operatorname{ker} \sigma$ and $\sigma=0$.

Case 2. $B$ is bounded and $\operatorname{Exp} B<\operatorname{Exp} A$. Since $B$ is bounded, it is a direct sum of cyclic groups; let $[B]$ be a basis of $B$. Since $\operatorname{Exp} B \leqq \operatorname{Exp} A$, for all $b \in[B]$ there exists a homomorphism $\tau_{b}: B \rightarrow A$ such that $o(b)=o\left(b \tau_{b}\right)$ and $b^{\prime} \tau_{b}=0$ for all $b^{\prime} \in[B]$ with $b^{\prime} \neq b$. Then we have

$$
0=\bigcap_{b \in[B]} \operatorname{ker} \tau_{b}=\bigcap_{\tau: B \rightarrow A} \operatorname{ker} \tau,
$$

and from (2) it follows that $A \sigma=0$ and $\sigma=0$. Q.E.D.

Proposition 1.8. Let $A$ be a reduced Abelian p-group, let $\{a\}$ be a direct summand of $A$ and let $x$ be an element of $A$ with $o(x) \leqq o(a)$. Then there exist an integer $n$ and an automorphism $\gamma$ of $A$ such that $x=n a+a \gamma$.

Proof. Let $A=\{a\} \oplus C$ and let $x=n^{\prime} a+c$ with $c \in C$. Let $\sigma$ be defined by $a \sigma=c$ and $C \sigma=0$; since $o(c) \leqq o(x) \leqq o(a), \sigma$ is an endomorphism of $A$. Since $\sigma^{2}=0$, $\gamma=1+\sigma$ is an automorphism of $A$. Let $n=n^{\prime}-1$; then $x=n a+a \gamma$. Q.E.D.

LEMMA 1.9. Let $A$ and $B$ be reduced Abelian $p$-groups with $p \neq 2$ and let $\operatorname{Hom}(A, B)$ $=\Delta \oplus \Lambda$, where both $\Delta$ and $\Lambda$ are invariant under Aut $A$ and Aut $B$. Then it follows that $\Delta=0$ or $\Lambda=0$.

We remark here that $\operatorname{Hom}(A, B)$ is a (End $A$-End $B$ )-bimodule.

Proof. Let us assume that $\Delta \neq 0 \neq \Lambda$; then there are three cases to consider:

Case 1. $A \Delta \subseteq p^{\omega} B$. Let $\sigma \in \operatorname{Hom}(A, B)$ with $A \sigma \subseteq\{b\}$, where $\{b\}$ is a direct summand of $B$, and let $B=\{b\} \oplus B^{\prime}$. Let the involution $\alpha \in$ Aut $B$ be defined by 
$\{b\}=B_{+}^{\alpha}$ and $B^{\prime}=B_{-}^{\alpha}$; then $\sigma \alpha=\sigma$. Since Hom $(A, B)=\Delta \oplus \Lambda$, there exist elements $\delta \in \Delta$ and $\lambda \in \Lambda$ such that $\sigma=\delta+\lambda$, and this implies $2 \sigma=\sigma(\alpha+1)=\delta(\alpha+1)+\lambda(\alpha+1)$. Now $A \delta \subseteq p^{\omega} B \subseteq B^{\prime}$ and this implies $\delta(\alpha+1)=0$. Hence, $\sigma=2^{-1} \lambda \alpha+2^{-1} \lambda \in \Lambda$, and we have shown that

$(+)$ if $\sigma \in \operatorname{Hom}(A, B)$ such that $A \sigma$ is contained in a cyclic direct summand of $B$, then $\sigma \in \Lambda$.

Let $0 \neq \delta \in \Delta$. By Proposition 1.8, $A$ is generated by cyclic direct summands; hence, there exists a cyclic direct summand $\{a\}$ of $A$ such that $a \delta \neq 0$. Let $A=\{a\} \oplus A^{\prime}$ and define $\beta \in$ Aut $A$ by $\{a\}=A_{+}^{\beta}$ and $A^{\prime}=A_{-}^{\beta}$; then $\delta^{\prime}=(\beta+1) \delta \in \Delta$, and we have

$$
A \delta^{\prime}=\left\{a \delta^{\prime}\right\} \neq 0 \text { and } A^{\prime} \delta^{\prime}=0 .
$$

Let $\{b\}$ be a direct summand of $B$ with $o(b) \geqq o\left(a \delta^{\prime}\right)$, let $p^{i}=o(b) / o\left(a \delta^{\prime}\right)$ and let $b^{\prime}=p^{i} b$; then we have $o\left(b^{\prime}\right)=o\left(a \delta^{\prime}\right)$. Define $\sigma \in \operatorname{Hom}(A, B)$ by $a \sigma=b^{\prime}$ and $A^{\prime} \sigma=0$; then $\sigma \in \Lambda$ by $(+)$, and since $A \delta^{\prime} \subseteq p^{\omega} B$, there exists an element $x \in B$ such that $a \delta^{\prime}=p^{i} x$. Then $o(x)=o(b)$ and from Proposition 1.8 we get $x=n b+b \gamma$ for some $\gamma \in$ Aut $B$. Hence, $\sigma(n+\gamma) \in \Lambda$ and

$$
a \sigma(n+\gamma)=b^{\prime}(n+\gamma)=p^{i} b(n+\gamma)=p^{i} x=a \delta^{\prime} .
$$

Thus we get $0 \neq \sigma(n+\gamma)=\delta^{\prime} \in \Delta \cap \Lambda$, a contradiction.

Case 2. $A \Lambda \subseteq p^{\omega} B$. The same argument as in Case 1 yields the contradiction $\Delta \cap \Lambda \neq 0$.

Case 3. $A \Delta \nsubseteq p^{\omega} B$ and $A \Lambda \nsubseteq p^{\omega} B$. Let $\delta \in \Delta$ and $\lambda \in \Lambda$ such that $A \delta \nsubseteq p^{\omega} B$ and $A \lambda \nsubseteq p^{\omega} B$. Let $a \in A$ such that $a \delta \notin p^{\omega} B$. By Lemma 1.2, there exists a direct summand $\{b\}$ of $B, B=\{b\} \oplus B^{\prime}$, such that $a \delta=b^{*}+b^{\prime}$, where $0 \neq b^{*} \in\{b\}$ and $b^{\prime} \in B^{\prime}$. Let $\beta \in$ Aut $B$ be defined by $\{b\}=B_{+}^{\beta}$ and $B^{\prime}=B_{-}^{\beta}$; then $\delta^{\prime}=\delta(\beta+1) \in \Delta$, and we have $0 \neq A \delta^{\prime}=\left\{b^{*}\right\} \subseteq\{b\}$. Let $\delta^{\prime \prime}$ be a multiple of $\delta^{\prime}$ with $o\left(\delta^{\prime \prime}\right)=p$; then $A \delta^{\prime \prime}$ has order $p$ and it is contained in a cyclic direct summand of $B$. Applying the same argument to $\lambda$, we get

(1) There exist elements $\delta \in \Delta$ and $\lambda \in \Lambda$ of order $p$, such that $A \delta$ and $A \lambda$ are contained in some (not necessarily the same) cyclic direct summand of $B$.

Let $\left\{b_{1}\right\}$ and $\left\{b_{2}\right\}$ be direct summands of $B$ with $A \delta \subseteq\left\{b_{1}\right\}$ and $A \lambda \subseteq\left\{b_{2}\right\}$. Clearly we may assume that $o\left(b_{1}\right) \leqq o\left(b_{2}\right)$; let $b_{2}^{*}=\left[o\left(b_{2}\right) / o\left(b_{1}\right)\right] b_{2}$. Then $o\left(b_{2}^{*}\right)=o\left(b_{1}\right)$, and by Proposition 1.8 there exist $\gamma \in$ Aut $B$ and an integer $n$ such that $b_{2}^{*}=n b_{1}+b_{1} \gamma$. Let $\delta^{\prime}=\delta(n+\gamma) \in \Delta$; then $A \delta^{\prime} \neq 0$ since $o\left(b_{2}^{*}\right)=o\left(b_{1}\right)$ and

$$
A \delta^{\prime}=A \delta(n+\gamma) \subseteq\left\{b_{1}\right\}(n+\gamma)=\left\{b_{2}^{*}\right\} \subseteq\left\{b_{2}\right\} .
$$

We conclude that

(2) There exist elements $\delta \in \Delta$ and $\lambda \in \Lambda$ of order $p$, such that $A \delta$ and $A \lambda$ are contained in the same cyclic direct summand of $B$.

Let $A \delta \subseteq\{b\}$ and $A \lambda \subseteq\{b\}$, where $\{b\}$ is a direct summand of $B$. By Proposition 1.8, $A$ is generated by its cyclic direct summands; let $\left\{a_{1}\right\}$ and $\left\{a_{2}\right\}$ be direct summands of $A$ such that $a_{1} \delta \neq 0 \neq a_{2} \lambda$, and assume that $o\left(a_{1}\right) \leqq o\left(a_{2}\right)$. By Proposition 
1.8 , there exist $\gamma \in$ Aut $A$ and an integer $n$ such that $a_{1}=n a_{2}+a_{2} \gamma$. Then we have $(n+\gamma) \delta \in \Delta$ and $a_{2}(n+\gamma) \delta=a_{1} \delta \neq 0$, and we conclude that

(3) There exist elements $\delta \in \Delta$ and $\lambda \in \Lambda$ of order $p$, a direct summand $\{a\}$ of $A$ and a direct summand $\{b\}$ of $B$, such that $a \delta \neq 0 \neq a \lambda$ and $A \delta \subseteq\{b\}$ and $A \lambda \subseteq\{b\}$.

Let $A=\{a\} \oplus A^{\prime}$ and let $\alpha \in$ Aut $A$ be defined by $\{a\}=A_{+}^{\alpha}$ and $A^{\prime}=A_{-}^{\alpha}$. Then $\delta^{\prime}=(\alpha+1) \delta \in \Delta$ and $\lambda^{\prime}=(\alpha+1) \lambda \in \Lambda$, and $A^{\prime} \delta^{\prime}=0=A^{\prime} \lambda^{\prime}$. Since $\{a\} \delta^{\prime}=\{a\} \lambda^{\prime}$, there exists an integer $n$ with $\delta^{\prime}=n \lambda^{\prime}$, and this says $0 \neq \delta^{\prime}=n \lambda^{\prime} \in \Delta \cap \Lambda$, a contradiction. This completes the proof.

Definition. A reduced Abelian group $A$ is a ( $p p$ )-group if and only if $A \neq 0$ and $A$ is a $p$-group such that $A$ has two independent elements of maximal order if $A$ is bounded.

Evidently, the reduced Abelian $p$-group $A \neq 0$ is a $(p p)$-group if and only if whenever $A=\{u\} \oplus C$, then $C=\{x\} \oplus D$ with $o(u) \leqq o(x)$.

Proposition 1.10. Let $A$ be a reduced Abelian ( $p p)$-group with $p \neq 2$, let $\{u\}$ be a direct summand of $A$ and let $\gamma$ be an automorphism of $A[o(u)]$. Then the following are equivalent:

(a) $P_{\gamma}=P$ for all cyclic direct summands $P$ of $A$ with $|P| \leqq o(u)$.

(b) There exists an integer $n$ such that $x \gamma=n x$ for all $x \in A[o(u)]$.

Proof. Since the implication (b) $\Rightarrow$ (a) is obvious, we need only to show that (a) implies (b).

Let $o(u)=p^{k}$ and let $A=\{u\} \oplus C$; then $A\left[p^{k}\right]=\{u\} \oplus C\left[p^{k}\right]$, and since $A$ is a (pp)-group, $C\left[p^{k}\right]$ is generated by elements of order $p^{k}$. Let $x \in C\left[p^{k}\right]$ with $o(x)=p^{k}$. Then $\{u\},\{u+x\}$ and $\{u-x\}$ are direct summands of order $p^{k}$ of $A$, and hence, there exist integers $n, m$ and $m^{\prime}$ such that

$$
u \gamma=n u, \quad(u+x) \gamma=m(u+x) \text { and }(u-x) \gamma=m^{\prime}(u-x) .
$$

It follows that

$$
2 n u=\left(m+m^{\prime}\right) u+\left(m-m^{\prime}\right) x
$$

and since $o(u)=o(x)=p^{k}$, this implies $2 n \equiv m+m^{\prime}$ and $m \equiv m^{\prime} \bmod p^{k}$. Thus $2 n \equiv 2 m \equiv 2 m^{\prime} \bmod p^{k}$; but $p \neq 2$, and hence, $n \equiv m \equiv m^{\prime} \bmod p^{k}$. Consequently, $x y=n x$ for all $x \in C\left[p^{k}\right]$ with $o(x)=p^{k}$; and since $C\left[p^{k}\right]$ is generated by elements of order $p^{k}$, we get $x \gamma=n x$ for all $x \in A\left[p^{k}\right]$. Q.E.D.

Corollary 1.11. Let $A$ be a reduced Abelian ( $p p)$-group with $p \neq 2$ and let $\gamma$ be an automorphism of $A$. Then the following are equivalent:

(a) $\gamma \in c$ Aut $A$,

(b) $P \gamma=P$ for all cyclic direct summands $P$ of $A$,

(c) $U \gamma=U$ for all subgroups $U$ of $A$.

Proof. Let $\gamma \in c$ Aut $A$. Then $\gamma$ centralizes every extreme involution of $A$, and by Propositions 1.6 and 1.3(b) we get $P \gamma=P$ for all cyclic direct summands $P$ of $A$. Thus (a) implies (b). 
Assume that $\gamma$ satisfies (b) and let $U$ be a cyclic subgroup of $A$. Then $U$ lies in some $A\left[p^{k}\right]$, and from Proposition 1.10 we infer that $U \gamma=U$. Thus we have shown (c) for all cyclic subgroups of $A$; and since every subgroup is generated by its cyclic subgroups, this implies (c). Now (c) implies (b) trivially and (b) implies (a) by Proposition 1.10. Q.E.D.

\section{Remarks on Hom and duality.}

Notation. $t A$ is the maximal torsion subgroup of the Abelian group $A$.

Lemma 2.1. Let $A$ and $C$ be Abelian p-groups and let $B$ be a basic subgroup of $A$. Then $t$ Hom $(A, C)$ is isomorphic to $t \operatorname{Hom}(B, C)$.

Proof. Since torsion homomorphisms are small, from [9, (4.1), p. 233] we get that for every $\sigma \in t \operatorname{Hom}(B, C)$ there exists a unique small homomorphism $\sigma^{\psi}: A \rightarrow C$ extending $\sigma$; and it is an immediate consequence that $\psi$ maps $t$ Hom $(B, C)$ isomorphically onto $t \operatorname{Hom}(A, C)$.

Definition. Let $A$ be a reduced Abelian $p$-group. Then the adjoint group of $A$ is $A^{*}=t \operatorname{Hom}\left(A, Z\left(p^{\infty}\right)\right)$.

LEMmA 2.2. The following properties of the reduced Abelian p-group $A$ are equivalent:

(a) $A \simeq A^{*}$.

(b) $A \simeq A^{* *}$.

(c) $A \simeq t \sum_{0<i<\omega}^{*} B_{i}$, where each $B_{i}$ is a finite direct sum of cyclic groups of order $p^{i}$.

(d) $A$ is torsion complete and every Ulm-invariant of $A$ is finite.

Proof. Let $A \simeq A^{*}$; then $A^{*} \simeq A^{* *}$ and thus (a) implies (b). Let $A \simeq A^{* *}$ and let $B=\sum_{0<i<\omega}^{\circ} B_{i}$ be a basic subgroup of $A$, where each $B_{i}$ is a direct sum of cyclic groups of order $p^{i}$. By Lemma 2.1, we get

$$
A^{*} \simeq t \operatorname{Hom}\left(\sum_{0<i<\omega}^{\circ} B_{i}, Z\left(p^{\infty}\right)\right) \simeq t \sum_{0<i<\omega}^{*} B_{i}^{*} .
$$

Hence, basic subgroups of $A^{*}$ are isomorphic to $\sum_{0<i<\omega}^{\circ} B_{i}^{*}$; cf. Fuchs [4, Theorem 29.6 , p. 100]. By Lemma 2.1, we get

$$
A \simeq A^{* *} \simeq t \sum_{0<i<\omega}^{*} B_{i}^{* *} .
$$

Hence, basic subgroups of $A$ are isomorphic to $\sum_{0<i<\omega}^{\circ} B_{i}^{* *}$, and thus we have $B_{i} \simeq B_{i}^{* *}$ for all $i$. Now assume that $\left|B_{i}\right|=r \geqq \aleph_{0}$ for some $i$. Since $B_{i}$ is a direct sum of pairwise isomorphic cyclic groups, this implies $\left|B_{i}^{* *}\right|=2^{2^{r}}>r$, contradicting $B_{i} \simeq B_{i}^{* *}$. Hence, each $B_{i}$ is finite and we have shown that (b) implies (c).

Now if $A$ satisfies (c), then $\sum_{0<i<\omega}^{\circ} B_{i}$ is isomorphic to a basic subgroup of $A$, and this implies

$$
A^{*} \simeq t \operatorname{Hom}\left(\sum_{0<i<\omega}^{0} B_{i}, Z\left(p^{\infty}\right)\right) \simeq t \sum_{0<i<\omega}^{*} B_{i}^{*} .
$$


Since every $B_{i}$ is finite, we have $B_{i} \simeq B_{i}^{*}$ for all $i$; hence, $A \simeq A^{*}$ and we have shown that (c) implies (a). Now the equivalence of (c) and (d) follows from [4, Theorem 34.1 , p. 114], completing the proof.

Definition. Let $\sigma: A \rightarrow B$ be a homomorphism of reduced Abelian $p$-groups. Then the adjoint of $\sigma$ is the homomorphism $\sigma^{*}: B^{*} \rightarrow A^{*}$ defined by $f \sigma^{*}=\sigma f$ for all $f \in B^{*}$.

Of course, the mapping $*: \sigma \rightarrow \sigma^{*}$ has the usual properties, namely $(\sigma+\tau)^{*}$ $=\sigma^{*}+\tau^{*},(\sigma \tau)^{*}=\tau^{*} \sigma^{*},\left(1_{A}\right)^{*}=1_{A^{*}}$, whenever $\sigma+\tau$ and $\sigma \tau$ are defined.

Definition. Let $A$ be a reduced Abelian $p$-group. Then the mapping $\nu_{A}: A \rightarrow A^{* *}$ is defined by $f\left(x v_{A}\right)=x f$ for all $x \in A$ and all $f \in A^{*}$.

Obviously, $v_{A}$ is a homomorphism.

Proposition 2.3. Let $\sigma: A \rightarrow B$ be a homomorphism of reduced Abelian p-groups. Then we have $\sigma \nu_{B}=\nu_{A} \sigma^{* *}$.

The proof is left to the reader.

Definition. Let $A$ be a reduced Abelian $p$-group and let $A^{*}$ be its adjoint group. If $U \subseteq A$, then $U^{\times}=\left[f \in A^{*} \mid U f=0\right]$ is the annihilator of $U$ in $A^{*}$, and if $V \subseteq A^{*}$, then $V^{\times}=[x \in A \mid x V=0]$ is the annihilator of $V$ in $A$.

Proposition 2.4. Let $A$ be a reduced Abelian p-group and let $U \subseteq A$ such that $p^{\omega}(A / U)=0$. Then we have $U^{\times \times}=U$.

REMARK. We have $p^{\omega}(A / U)=0$ if and only if $U$ is closed in the $p$-adic topology on $A$.

Proof. Clearly we have $U \subseteq U^{\times \times}$; let us assume that $U \subset U^{\times \times}$. Then there exists an element $x \in U^{\times \times}$with $x \notin U$ and $p x \in U$; that is, $x+U$ is an element of order $p$ in $A / U$. Hence, $x+U$ is contained in a cyclic direct summand of $A / U-\mathrm{cf}$. Fuchs [5, top of p. 80]-and thus there exists an element $f^{\prime} \in(A / U)^{*}$ with $(x+U) f^{\prime}$ $\neq 0$. Let $c: A \rightarrow A / U$ be the canonical epimorphism and let $f=c f^{\prime}$. Then $f \in U^{\times}$ and, since $x \in U^{\times \times}$, we get

$$
0=x f=x\left(c f^{\prime}\right)=(x+U) f^{\prime} \neq 0,
$$

a contradiction.

Corollary 2.5. Let $A$ be a reduced Abelian p-group with $p^{\omega} A=0$ and let $U \subseteq A$. Then $U=U^{\times \times}$whenever $U$ is a finite or cofinite subgroup or a direct summand of $A$.

Proposition 2.6. Let $A$ be a reduced Abelian p-group and let $X$ be a finite set of subgroups of $A$ such that $0=\bigcap_{\mathrm{Y} \in X} Y$ and $A=Y+Y^{\prime}$ for all $Y \in X$, where $Y^{\prime}$ $=\bigcap_{Y \neq Z \in X} Z$. Then we have $A^{*}=\sum_{Y \in X}^{\circ} Y^{\times}$.

Proof. Let $X=\left[X_{1}, \ldots, X_{n}\right]$ and let $X_{i}^{\prime}=\bigcap_{j \neq i} X_{j}$ for all $i$. Then it follows by standard arguments that $A=\sum_{i}^{\circ} X_{i}^{\prime}$ and $X_{j}=\sum_{i \neq j}^{\circ} X_{i}^{\prime}$ for all $j$, and the proposition follows immediately.

Notation. If $X \subseteq A$, then $X^{\circ}$ is the annihilator of $X^{\times}$in $A^{* *}$. 
Proposition 2.7. Let $A$ be a reduced Abelian p-group and let $A=D \oplus E$. Then $A^{* *}=D^{\circ} \oplus E^{\circ}$, and we have $D^{\circ} \simeq D^{* *}$ and $D v_{A} \subseteq D^{\circ}$.

The straightforward proof is left to the reader.

Lemma 2.8. Let $A$ be a reduced Abelian p-group such that $A \simeq A^{*}$. Then $\nu_{A}$ is an isomorphism of $A$ onto $A^{* *}$.

Proof. Let $B=\sum_{0<i<\omega}^{\circ} B_{i}$ be a basic subgroup of $A$, where each $B_{i}$ is a direct sum of cyclic groups of order $p^{i}$. By Lemma 2.2, each $B_{i}$ is finite, and since $\nu_{A}$ is monomorphic by Lemma 1.2, we get $B_{i} \nu_{A}=B_{i}^{\circ} \simeq B_{i}^{* *} /$ for every $i$, by Proposition 2.7. For every $n$, let $A_{n} \subseteq A$ with $A=B_{1} \oplus \cdots \oplus B_{n} \oplus A_{n}$. From Lemma 2.2 and Proposition 2.7 it follows that $A^{* *}=\left(B_{1} \oplus \cdots \oplus B_{n}\right)^{\circ} \oplus A_{n}^{\circ}=B_{1}^{\circ} \oplus \cdots \oplus B_{n}^{\circ} \oplus A_{n}^{\circ}$ for all $n$, and since $B_{i} \simeq B_{i}^{\circ}$ is finite for every $i$, it follows that $B v_{A}=\sum_{i}^{\circ} B_{i} \nu_{A}=\sum_{i}^{\circ} B_{i}^{\circ}$ is a basic subgroup of $A^{* *}-\mathrm{cf}$. [5, Theorem 33.2, p. 140]. Hence, $B v_{A}$ is dense in $A^{* *}$; moreover, every element of $A^{* *}$ is limit of a bounded Cauchy sequence in $B v_{A}-\mathrm{cf}$. [7, Lemma 10] - and since $A$ is torsion complete by Lemma 2.2, it follows that $\nu_{A}$ is epimorphic, completing the proof.

Proposition 2.9. Let $A$ be a reduced Abelian p-group such that $A \simeq A^{*}$. Then we have $\nu_{A^{*}}\left(\nu_{A}\right)^{*}=1_{A^{*}}$ and $\left(\nu_{A}\right)^{*} \nu_{A^{*}}=1_{A^{*} \cdots}$.

Proof. (i) For all $h \in A^{*}$ and for all $x \in A$, we have

$$
x\left[h\left(v_{A^{*}}\left[v_{A}\right]^{*}\right)\right]=x\left[\left(h v_{A^{*}}\right)\left(\nu_{A}\right)^{*}\right]=x\left[v_{A}\left(h v_{A^{*}}\right)\right]=\left(x v_{A}\right)\left(h \nu_{A^{*}}\right)=h\left(x v_{A}\right)=x h ;
$$

that is, $\nu_{A^{*}}\left(\nu_{A}\right)^{*}=1_{A^{*}}$.

(ii) For all $g \in A^{* * *}$ and all $e \in A^{* *}$, we have

$$
e\left[g\left(\left(v_{A}\right)^{*} \nu_{A^{*}}\right)\right]=e\left[\left(g\left[\nu_{A}\right]^{*}\right) \nu_{A^{*}}\right]=\left(g\left[\nu_{A}\right]^{*}\right) e=\left(\nu_{A} g\right) e .
$$

Now $e=x \nu_{A}$ for some $x \in A$ by Lemma 2.8, and this implies

$$
e\left[g\left(\left(\nu_{A}\right) v_{A^{*}}\right)\right]=\left(\nu_{A} g\right)\left(x \nu_{A}\right)=x\left(\nu_{A} g\right)=e g .
$$

Hence, $\left(v_{A}\right) v_{A^{*}}=1_{A^{*}}$ Q. Q.E.D.

Lemma 2.10. Let $A$ be a reduced Abelian p-group such that $A \simeq A^{*}$. Then the mapping $\sigma \rightarrow \sigma^{*}$ is an anti-isomorphism of End $A$ onto End $A^{*}$.

Proof. Let $\sigma \in$ End $A$ such that $\sigma^{*}=0$. Then we have $f \sigma^{*}=\sigma f=0$ for all $f \in A^{*}$ and thus $x(\sigma f)=(x \sigma) f=0$ for all $x \in A$ and all $f \in A^{*}$. By Lemma 1.2, we get $A \sigma \subseteq p^{\omega} A=0$ and $\sigma=0$; that is, $*$ is one-to-one.

Let $\tau \in$ End $A^{*}$ and let $\sigma \in$ End $A$ be defined by $\sigma=\nu_{A} \tau^{*} \nu_{A}^{-1}$. Then we have $\sigma^{*}=\left(\nu_{A}^{*}\right)^{-1} \tau^{* *} \nu_{A}^{*}=\nu_{A^{*}} \tau^{* *}\left(\nu_{A^{*}}\right)^{-1}$ by Proposition 2.9, and from Proposition 2.3 we get $\sigma^{*}=\tau$. Thus $*$ is onto; and clearly $(\sigma+\tau)^{*}=\sigma^{*}+\tau^{*}$ and $(\sigma \tau)^{*}=\tau^{*} \sigma^{*}$ for all $\sigma, \tau \in$ End $A$, completing the proof.

Definition. Let $A$ be a reduced Abelian $p$-group such that $A \simeq A^{*}$. Then, by the preceding lemma, $*$ is an anti-isomorphism of End $A$ onto End $A^{*}$ and 
hence, * induces an anti-isomorphism of Aut $A$ onto Aut $A^{*}$. We define the isomorphism $\theta_{A}$ : Aut $A \rightarrow$ Aut $A^{*}$ by $\gamma^{\theta_{A}}=\left(\gamma^{*}\right)^{-1}$ for all $\gamma \in$ Aut $A$.

Proposition 2.11. Let $A$ be a reduced Abelian p-group such that $A \simeq A^{*}$. Then we have $\left(\Sigma_{A} H\right)^{\theta_{A}}=\Sigma_{A} \cdot H^{\times}$whenever $H=H^{\times \times}$.

Proof. Let $\gamma=1+\sigma \in \Sigma_{A} H$. Then we have $A \sigma \subseteq H$ and $H \sigma=0$ and this implies $(A \sigma) H^{\times}=A\left(\sigma H^{\times}\right)=A\left(H^{\times} \sigma^{*}\right)=0$ and $(H \sigma) A^{*}=H\left(\sigma A^{*}\right)=H\left(A^{*} \sigma^{*}\right)=0$; hence, $H^{\times} \sigma^{*}=0$ and $A^{*} \sigma^{*} \subseteq H^{\times}$. Thus $\gamma^{*}=1+\sigma^{*} \in \Sigma_{A^{*}} H^{\times}$and we have shown that $\left(\Sigma_{A} H\right)^{*} \subseteq \Sigma_{A^{*}} H^{\times}$.

Let $\delta=1+\tau \in \Sigma_{A^{*}} H^{\times}$. By Lemma 2.10, we have $\tau=\sigma^{*}$ for some $\sigma \in$ End $A$. Thus $H^{\times} \sigma^{*}=0$ and $A^{*} \sigma^{*} \subseteq H^{\times}$, that is, $A\left(H^{\times} \sigma^{*}\right)=A\left(\sigma H^{\times}\right)=(A \sigma) H^{\times}=0$ and $H\left(A^{*} \sigma^{*}\right)=(H \sigma) A^{*}=0$. Hence, $A \sigma \subseteq H^{\times \times}=H$ by hypothesis and $H \sigma \subseteq p^{\omega} A=0$ by Lemma 1.2. Thus $\gamma=1+\sigma \in \Sigma_{A} H$ and $\gamma^{*}=\delta$, and we have shown that $\left(\Sigma_{A} H\right)^{*}$ $=\Sigma_{A^{*}} H^{\times}$. Now $\left(\Sigma_{A} H\right)^{*}=\left(\Sigma_{A} H\right)^{\theta}$, and this completes the proof.

\section{Stabilizers of direct summands.}

Proposition 3.1. Let $A$ be a reduced Abelian p-group with $p>3$, let $\Gamma=$ Aut $A$ and let $\delta$ and $\alpha$ be automorphisms of $A$ such that

(1) $\alpha^{2}=1$,

(2) $(\alpha \delta)^{2}=1$,

(3) $\delta \delta^{\gamma}=\delta^{\gamma} \delta$ for all $\gamma \in c c_{\Gamma}\{\alpha\}$.

Then there exists an involution $\beta$ of $A$ with $\alpha \beta=\beta \alpha$ such that

(a) $(\delta-\beta)^{2}=0$,

(b) $A_{+}^{\alpha}(\delta-\beta) \subseteq A_{-}^{\alpha}$ and $A_{-}^{\alpha}(\delta-\beta) \subseteq A_{+}^{\alpha}$.

Proof. If $\alpha= \pm 1$, then (2) implies $\delta^{2}=1$ and $\beta=\delta$ has properties (a) and (b). Let $\alpha \neq \pm 1$. Then $A_{+}^{\alpha} \neq 0$ and $A_{-}^{\alpha} \neq 0$ and we have $A=A_{+}^{\alpha} \oplus A_{-}^{\alpha}$ by Proposition 1.3(a).

The ring of all endomorphisms of $A$ is isomorphic to the ring of all matrices $\left(\begin{array}{ll}\lambda & \sigma \\ \tau & \rho\end{array}\right)$, where $\lambda \in \operatorname{Hom}\left(A_{+}^{\alpha}, A_{+}^{\alpha}\right), \quad \sigma \in \operatorname{Hom}\left(A_{+}^{\alpha}, A_{-}^{\alpha}\right), \quad \tau \in \operatorname{Hom}\left(A_{-}^{\alpha}, A_{+}^{\alpha}\right)$ and $\rho \in \operatorname{Hom}\left(A_{-}^{\alpha}, A_{-}^{\alpha}\right)$; we shall regard this isomorphism as an identification. Thus $\alpha=\left(\begin{array}{ll}1 & -0 \\ 0 & -1\end{array}\right)$ and the elements of $c_{\Gamma}\{\alpha\}$ are, by Proposition 1.3(b), the matrices $\left(\begin{array}{ll}\xi \\ 0\end{array}\right)$, where $\xi \in$ Aut $A_{+}^{\alpha}$ and $\eta \in$ Aut $A_{-}^{\alpha}$.

Let $\delta=\left(\begin{array}{cc}\lambda & \sigma \\ \tau & \rho\end{array}\right)$ and let $\gamma=\left(\begin{array}{cc}\xi & 0 \\ 0 & \eta\end{array}\right) \in c_{\Gamma}\{\alpha\}$; then we have

$$
\delta^{\gamma}=\left(\begin{array}{ll}
\xi^{-1} \lambda \xi & \xi^{-1} \sigma \eta \\
\eta^{-1} \tau \xi & \eta^{-1} \rho \eta
\end{array}\right) .
$$

Hence, from (2) it follows that $\delta^{-1}=\alpha \delta \alpha=\left(\begin{array}{cc}\lambda & -\sigma \\ -\mathfrak{\tau} & \rho\end{array}\right)$ and this implies

$$
1=\left(\begin{array}{ll}
1 & 0 \\
0 & 1
\end{array}\right)=\left(\begin{array}{cc}
\lambda^{2}-\sigma \tau & \lambda \sigma-\sigma \rho \\
\rho \tau-\tau \lambda & \rho^{2}-\tau \sigma
\end{array}\right)
$$


Hence,

$$
1=\lambda^{2}-\sigma \tau \text { and } 1=\rho^{2}-\tau \sigma .
$$

Let $\gamma \in c c_{\Gamma}\{\alpha\}$. Then $\delta \delta^{\gamma}=\delta^{\gamma} \delta$ by (3), and a straightforward computation shows that

$$
\xi^{-1} \lambda \xi \lambda+\xi^{-1} \sigma \eta \tau=\lambda \xi^{-1} \lambda \xi+\sigma \eta^{-1} \tau \xi
$$

and

$(++)$

$$
\eta^{-1} \tau \xi \sigma+\eta^{-1} \rho \eta \rho=\tau \xi^{-1} \sigma \eta+\rho \eta^{-1} \rho \eta
$$

Now let $\eta=1$; then, by $\left({ }^{*}\right)$, we get

$$
\lambda \xi \lambda+\lambda^{2}-1=\xi \lambda \xi^{-1} \lambda \xi+\xi\left(\lambda^{2}-1\right) \xi
$$

from $(+)$ and from $(++)$ we get with $\xi=1$

$\left(+++^{\prime}\right)$

$$
\rho^{2}-1+\rho \eta \rho=\eta\left(\rho^{2}-1\right) \eta+\eta \rho \eta^{-1} \rho \eta \text {. }
$$

Now let $\xi=2$ in $\left(t^{\prime}\right)$ and $\eta=2$ in $\left(++^{\prime}\right)$. Then $\left(t^{\prime}\right)$ becomes $3 \lambda^{2}-1=6 \lambda^{2}-4$ and $\left(++^{\prime}\right)$ becomes $3 \rho^{2}-1=6 \rho^{2}-4$; thus $3=3 \lambda^{2}$ and $3=3 \rho^{2}$, and since $p>3$, this implies $\lambda^{2}=1$ and $\rho^{2}=1$; and from $\left(^{*}\right)$ we infer that

$$
\sigma \tau=0 \text { and } \tau \sigma=0 .
$$

Let $\beta=\left(\begin{array}{ll}\lambda & 0 \\ 0 & \rho\end{array}\right)$; then $\beta^{2}=1$ and $\alpha \beta=\beta \alpha$ and $\delta-\beta=\left(\begin{array}{ll}0 & \sigma \\ \tau & 0\end{array}\right)$. From $(\circ)$ it follows that $(\delta-\beta)^{2}=0$, and clearly we have $A_{+}^{\alpha}(\delta-\beta) \subseteq A_{-}^{\alpha}$ and $A_{-}^{\alpha}(\delta-\beta) \subseteq A_{+}^{\alpha}$. Q.E.D.

Definition 3.2. Let $\Gamma$ be a group and let $\alpha \in \Gamma$ be an involution. Then $[\alpha]$ is the set of all subgroups $\Delta$ of $\Gamma$ such that

(1) $(\alpha \delta)^{2}=1$ for all $\delta \in \Delta$,

(2) $c_{\Gamma}\{\alpha\} \subseteq n_{\Gamma} \Delta$,

(3) the mapping $\delta \rightarrow \delta^{2}$ is an automorphism of $\Delta$.

Let $\Lambda$ be a subgroup of $\Gamma$. Then $\Lambda$ is $\alpha$-maximal if, and only if, $\Lambda \in[\alpha]$ and $\Lambda$ is maximal with respect to this property.

Let $\Lambda$ be a subgroup of $\Gamma$. Then $\Lambda$ is $\alpha$-indecomposable if, and only if, $\Lambda$ is normalized by $c_{\Gamma}\{\alpha\}$ and, whenever $\Lambda$ is a direct product of subgroups $\Delta_{1}$ and $\Delta_{2}$ which are normalized by $c_{\Gamma}\{\alpha\}$, it follows that $\Delta_{1}=1$ or $\Delta_{2}=1$.

REMARKs. (A) Every group in $[\alpha]$ is Abelian. (B) $[\alpha]$ is inductive. (C) If $\Delta \subseteq \Gamma$ is the direct product of its subgroups $\Delta_{1}$ and $\Delta_{2}$, then $\Delta_{1}, \Delta_{2} \in[\alpha]$ implies $\Delta \in[\alpha]$.

Lemma 3.3. Let $A$ be an Abelian p-group with $p>3$ and let $\alpha$ be an involution of $A$. Then the following properties of the subgroup $\Delta$ of $\Gamma=$ Aut $A$ are equivalent:

(1) $\Delta$ is $\alpha$-maximal.
(a) $\Delta=\left\{\Delta \cap \Sigma_{+}^{\alpha}, \Delta \cap \Sigma_{-}^{\alpha}\right\}$
(b) $c_{\Gamma}\{\alpha\} \subseteq n_{\Gamma}\left(\Delta \cap \Sigma_{+}^{\alpha}\right) \cap n_{\Gamma}\left(\Delta \cap \Sigma_{-}^{\alpha}\right)$,
(c) $\Sigma_{-}^{\alpha} \cap c_{\Gamma}\left(\Delta \cap \Sigma_{+}^{\alpha}\right)=\Delta \cap \Sigma_{-}^{\alpha}$,
(d) $\Sigma_{+}^{\alpha} \cap c_{\Gamma}\left(\Delta \cap \Sigma_{-}^{\alpha}\right)=\Delta \cap \Sigma_{+}^{\alpha}$. 
REMARK. In particular, every $\alpha$-maximal subgroup $\Delta$ of $\Gamma$ is the direct product of $\Delta \cap \Sigma_{+}^{\alpha}$ and $\Delta \cap \Sigma_{-}^{\alpha}$, and these are normalized by $c_{\Gamma}\{\alpha\}$.

Proof. Let $\Delta$ be an $\alpha$-maximal subgroup of $\Gamma$ and suppose that $\alpha= \pm 1$. Then from part (1) of Definition 3.2 we get $\delta^{2}=1$ for all $\delta \in \Delta$, and from part (3) of the same definition it follows that $\Delta=1$. Since $\Sigma_{+}^{\alpha}=1=\Sigma_{-}^{\alpha}$ for $\alpha= \pm 1$, we get (2(a))(2(d)).

Suppose that $\alpha \neq \pm 1$. Then by Proposition 3.1, every element $\delta \in \Delta$ has the form $\delta=\left(\begin{array}{cc}\lambda & \sigma \\ \tau & \rho\end{array}\right)$ with $\lambda \in \operatorname{Hom}\left(A_{+}^{\alpha}, A_{+}^{\alpha}\right), \sigma \in \operatorname{Hom}\left(A_{+}^{\alpha}, A_{-}^{\alpha}\right), \tau \in \operatorname{Hom}\left(A_{-}^{\alpha}, A_{+}^{\alpha}\right)$, $\rho \in \operatorname{Hom}\left(A_{-}^{\alpha}, A_{-}^{\alpha}\right)$ and

$$
\lambda^{2}=1, \quad \rho^{2}=1, \quad \sigma \tau=0 \text { and } \tau \sigma=0 .
$$

This implies $\delta^{2}=\left(\begin{array}{cc}1 & \sigma^{\prime} \\ \tau^{\prime} & 1\end{array}\right)$ with $\sigma^{\prime} \tau^{\prime}=0$ and $\tau^{\prime} \sigma^{\prime}=0$, and from $\Delta^{2}=\Delta$ it follows that

$(+)$ Every element $\delta \in \Delta$ has the form $\delta=\left(\begin{array}{ll}1 & \sigma \\ \tau & 1\end{array}\right)$ with $\sigma \tau=0$ and $\tau \sigma=0$.

Let

$$
\Delta_{+}=\left[\gamma \in \Sigma_{+}^{\alpha} \mid \gamma=\left(\begin{array}{ll}
1 & 0 \\
\tau & 1
\end{array}\right) \text { and }\left(\begin{array}{ll}
1 & \sigma \\
\tau & 1
\end{array}\right) \in \Delta \text { for some } \sigma\right]
$$

and

$$
\Delta_{-}=\left[\gamma \in \Sigma_{-}^{\alpha} \mid \gamma=\left(\begin{array}{ll}
1 & \sigma \\
0 & 1
\end{array}\right) \text { and }\left(\begin{array}{ll}
1 & \sigma \\
\tau & 1
\end{array}\right) \in \Delta \text { for some } \tau\right]
$$

A straightforward argument shows that $\Delta_{+}$and $\Delta_{-}$are subgroups of $\Sigma_{+}^{\alpha}$ and $\Sigma_{-}^{\alpha}$ respectively; and furthermore, we have $\Delta_{+} \in[\alpha]$ and $\Delta_{-} \in[\alpha]$. Now the subgroup $\Delta^{*}$ generated by $\Delta_{+}$and $\Delta_{-}$is the direct product of $\Delta_{+}$and $\Delta_{-}$, and this implies $\Delta^{*} \in[\alpha]$. Since $\Delta \subseteq \Delta^{*}$ and since $\Delta$ is $\alpha$-maximal, we get $\Delta=\Delta^{*}$, proving (2(a)) and (2(b)).

Let $\Delta_{+}^{*}=\Sigma_{+}^{\alpha} \cap c_{\Gamma} \Delta_{-}$and $\Delta_{-}^{*}=\Sigma_{-}^{\alpha} \cap c_{\Gamma} \Delta_{+} ;$then we have $\Delta_{+} \subseteq \Delta_{+}^{*}$ and $\Delta_{-} \subseteq \Delta_{-}^{*}$, and from $c_{\Gamma}\{\alpha\} \subseteq n_{\Gamma} \Delta_{+} \cap n_{\Gamma} \Delta_{-}$we infer $c_{\Gamma}\{\alpha\} \subseteq n_{\Gamma} c_{\Gamma} \Delta_{+} \cap n_{\Gamma} c_{\Gamma} \Delta_{-}$; thus $c_{\Gamma}\{\alpha\}$ $\subseteq n_{\Gamma} \Delta_{+}^{*}$ and $c_{\Gamma}\{\alpha\} \subseteq n_{\Gamma} \Delta_{-}^{*}$, and consequently we have

$$
\Delta_{+}^{*} \in[\alpha] \text { and } \Delta_{-}^{*} \in[\alpha] .
$$

Now the products $\Delta_{+}^{*} \Delta_{-}$and $\Delta_{+} \Delta_{-}^{*}$ are direct products, and this implies $\Delta_{+}^{*} \Delta_{-} \in[\alpha]$ and $\Delta_{+} \Delta_{-}^{*} \in[\alpha]$. Since $\Delta \subseteq \Delta_{+}^{*} \Delta_{-}$and $\Delta \subseteq \Delta_{+} \Delta_{-}^{*}$ and since $\Delta$ is $\alpha-$ maximal, we conclude that $\Delta=\Delta_{+} \Delta_{-}^{*}=\Delta_{+}^{*} \Delta_{-}$. Hence, $\Delta_{+}^{*}=\Delta_{+}$and $\Delta_{-}^{*}=\Delta_{-}$; we have proved (2(c)) and (2(d)) and thus (1) implies (2).

Let $\Delta$ be a subgroup of $\Gamma$ satisfying conditions (2(a))-(2(d)) and let $\Delta_{+}=\Delta \cap \Sigma_{+}^{\alpha}$ and $\Delta_{-}=\Delta \cap \Sigma_{-}^{\alpha}$; then from (2(b)) it follows that $\Delta_{+} \in[\alpha]$ and $\Delta_{-} \in[\alpha]$, and since $\Delta$ is the direct product of $\Delta_{+}$and $\Delta_{-}$[by (2(a)), (2(c)), (2(d))], we have $\Delta \in[\alpha]$.

Let $\Delta^{*}$ be an $\alpha$-maximal subgroup of $\Gamma$ containing $\Delta ; \Delta^{*}$ exists because $[\alpha]$ is inductive. Then we have

$$
\Delta_{+} \subseteq \Delta^{*} \cap \Sigma_{+}^{\alpha} \text { and } \Delta_{-} \subseteq \Delta^{*} \cap \Sigma_{-}^{\alpha}
$$


and application of (2(c)) and (2(d)) to $\Delta$ and $\Delta^{*}$ yields $\Delta_{+}=\Delta^{*} \cap \Sigma_{+}^{\alpha}$ and $\Delta_{-}$ $=\Delta^{*} \cap \Sigma_{-}^{\alpha}$, and thus we have $\Delta=\left\{\Delta_{+}, \Delta_{-}\right\}=\Delta^{*}$. Thus (2) implies (1) and (1) and (2) are equivalent. Q.E.D.

TheOREM 3.4. Let $A$ be a reduced Abelian p-group with $p>3$. Then the following properties of the subgroup $\Delta$ of $\Gamma=$ Aut $A$ are equivalent:

(a) $\Delta$ is the stabilizer of a direct summand of $A$.

(b) There exists an involution $\alpha \in \Gamma$ such that $\Delta$ is both $\alpha$-maximal and $\alpha$-indecomposable.

Proof. Let $D$ be a direct summand of $A$, let $\Delta=\Sigma_{A} D$ and let $\alpha$ be an involution of $A$ such that $D=A_{+}^{\alpha}$. Then we have $\Delta=\Sigma_{+}^{\alpha}$ and thus $\Delta$ satisfies conditions (2(a)), (2(b)) and (2(d)) of Lemma 3.3.

Let $\delta \in \Sigma_{-}^{\alpha} \cap c_{\Gamma} \Sigma_{+}^{\alpha}$ and let $\delta=\left(\begin{array}{ll}1 & \sigma \\ 0 & 1\end{array}\right)$; then we have $\delta \delta^{\prime}=\delta^{\prime} \delta$ for all $\delta^{\prime}=\left(\begin{array}{ll}1 & 0 \\ 1 & 1\end{array}\right) \in \Sigma_{+}^{\alpha}$, and a straightforward computation shows that $\sigma \tau=0$ and $\tau \sigma=0$ for all $\tau: A_{-}^{\alpha} \rightarrow A_{+}^{\alpha}$. By Proposition 1.7, we infer that $\sigma=0$ and $\delta=1$, that is, $\Sigma_{-}^{\alpha} \cap c_{\Gamma} \Sigma_{+}^{\alpha}=1$; and from Lemma 3.3 we conclude that $\Sigma_{+}^{\alpha}=\Delta$ is $\alpha$-maximal.

Let $\Delta$ be the direct product of its subgroups $\Delta_{1}$ and $\Delta_{2}$, where both $\Delta_{1}$ and $\Delta_{2}$ are normalized by $c_{\Gamma}\{\alpha\}$.

By Hausen [6, Hilfssatz 1.4], the mapping $\gamma \rightarrow \gamma-1$ induces an isomorphism of $\Delta=\Sigma_{A} A_{+}^{\alpha}$ onto Hom $\left(A_{-}^{\alpha}, A_{+}^{\alpha}\right)$. Hence, Hom $\left(A_{-}^{\alpha}, A_{+}^{\alpha}\right)=\Sigma_{1} \oplus \Sigma_{2}$, where

$$
\Sigma_{1}=\left(\Delta_{1}-1\right) \mid A_{-}^{\alpha} \text { and } \Sigma_{2}=\left(\Delta_{2}-1\right) \mid A_{-}^{\alpha} ;
$$

and since $\Delta_{1}$ and $\Delta_{2}$ are normalized by $c_{\Gamma}\{\alpha\}$, it follows that $\Sigma_{1}$ and $\Sigma_{2}$ are invariant under both Aut $A_{+}^{\alpha}$ and Aut $A_{-}^{\alpha}$. Hence $\Sigma_{1}=0$ or $\Sigma_{2}=0$ by Lemma 1.9; that is, $\Delta_{1}=1$ or $\Delta_{2}=1$. We have shown that $\Delta=\Sigma_{+}^{\alpha}$ is $\alpha$-indecomposable and thus (a) implies (b).

Let $\Delta$ be a subgroup of $\Gamma$ and let $\alpha \in \Gamma$ be an involution such that $\Delta$ is both $\alpha$-maximal and $\alpha$-indecomposable. By Lemma 3.3, $\Delta$ is the direct product of $\Delta \cap \Sigma_{+}^{\alpha}$ and $\Delta \cap \Sigma_{-}^{\alpha}$, and these are normalized by $c_{\Gamma}\{\alpha\}$. Hence, $\Delta \cap \Sigma_{+}^{\alpha}=1$ or $\Delta \cap \Sigma_{-}^{\alpha}=1$, that is, $\Delta \subseteq \Sigma_{+}^{\alpha}$ or $\Delta \subseteq \Sigma_{-}^{\alpha}$. Now $\Sigma_{+}^{\alpha} \in[\alpha]$ and $\Sigma_{-}^{\alpha} \in[\alpha]$, and since $\Delta$ is $\alpha$-maximal, it follows that $\Delta=\Sigma_{+}^{\alpha}$ or $\Delta=\Sigma_{-}^{\alpha}$. Thus (b) implies (a) and hence, (a) and (b) are equivalent.

Lemma 3.5. Let $A$ be a reduced Abelian p-group with $p>3$ and let $B$ and $C$ be elements of $H A$ such that $\Sigma_{A} B=\Sigma_{A} C$. Then $B=C$.

Proof. Let $\Gamma=$ Aut $A$ and let $\alpha$ and $\beta$ be involutions of $A$ such that $B=A_{+}^{\alpha}$ and $C=A_{+}^{\beta}$. Then $\Sigma_{+}^{\alpha}=\Sigma_{+}^{\beta}$ and this implies $\delta^{-1}=\alpha \delta \alpha=\beta \delta \beta$ for all $\delta \in \Sigma_{+}^{\alpha}$; that is, $\alpha \beta \in c_{\Gamma} \Sigma_{+}^{\alpha}$.

Let $\delta=\left(\begin{array}{ll}\lambda & \sigma \\ \tau & \rho\end{array}\right) \in c_{\Gamma} \Sigma_{+}^{\alpha}$. Then $\delta$ commutes with every $\left(\begin{array}{ll}1 & 0 \\ \tau^{\prime} & 1\end{array}\right) \in \Sigma_{+}^{\alpha}$, and a straightforward computation shows that $\sigma \tau^{\prime}=0$ and $\tau^{\prime} \sigma=0$ for all $\tau^{\prime}: A_{-}^{\alpha} \rightarrow A_{+}^{\alpha}$. From Proposition 1.7 we infer that $\sigma=0$, and we have shown that $c_{\Gamma} \Sigma_{+}^{\alpha} \subseteq \Sigma_{+}^{\alpha} c_{\Gamma}\{\alpha\}$. 
Thus we have $\alpha \beta=\delta \gamma$, where $\delta \in \Sigma_{+}^{\alpha}$ and $\gamma \in c_{\Gamma}\{\alpha\}$; and since $\delta \in \Sigma_{+}^{\alpha}$ it follows that $\gamma \in c_{\Gamma} \Sigma_{+}^{\alpha}$. Now we have

$$
\beta=\alpha \delta \gamma
$$

and hence, $1=(\alpha \delta \gamma)^{2}=(\alpha \delta) \gamma(\alpha \delta) \gamma=(\alpha \delta)(\alpha \gamma)(\delta \gamma)=(\alpha \delta)^{2} \gamma^{2}$; and since $(\alpha \delta)^{2}=1$, we get $\gamma^{2}=1$.

Since $\alpha \gamma=\gamma \alpha$, we have

$$
A=\left(A_{+}^{\alpha} \cap A_{+}^{\gamma}\right) \oplus\left(A_{+}^{\alpha} \cap A_{-}^{\gamma}\right) \oplus\left(A_{-}^{\alpha} \cap A_{+}^{\gamma}\right) \oplus\left(A_{-}^{\alpha} \cap A_{-}^{\gamma}\right)
$$

by Proposition 1.3(c). If $A_{+}^{\alpha} \cap A_{-}^{\gamma} \neq 0 \neq A_{-}^{\alpha} \cap A_{+}^{\gamma}$, then there exists an endomorphism $\sigma$ of $A$ such that $0 \neq\left(A_{-}^{\alpha} \cap A_{+}^{\gamma}\right) \sigma \subseteq A_{+}^{\alpha} \cap A_{-}^{\gamma}$ and $A_{+}^{\alpha} \sigma=0=\left(A_{-}^{\alpha} \cap A_{-}^{\gamma}\right) \sigma$. Then $1 \neq \delta=1+\sigma \in \Sigma_{+}^{\alpha}$ and $\delta^{\gamma}=1-\sigma=\delta^{-1}$; but $\gamma \in c_{\Gamma} \Sigma_{+}^{\alpha}$, and this implies $\delta=\delta^{-1}$ and $\delta^{2}=1$, a contradiction. Hence,

$$
A_{+}^{\alpha} \cap A_{-}^{\nu}=0 \text { or } A_{-}^{\alpha} \cap A_{+}^{\nu}=0
$$

and similarly it follows that

$$
A_{+}^{\alpha} \cap A_{+}^{\nu}=0 \text { or } A_{-}^{\alpha} \cap A_{-}^{\nu}=0 .
$$

Since $A_{+}^{\alpha} \neq 0 \neq A_{-}^{\alpha}$, from (*) it follows that $\gamma=1$ or $\gamma=-1$; and from (+) we infer that $\beta=\alpha \delta$ or $\beta=-\alpha \delta$, where $\delta \in \Sigma_{+}^{\alpha}$.

Let $x \in A_{+}^{\alpha}$. Then we have $x=x \alpha$ and $x \alpha \delta=x \delta=x$, and we have shown that $A_{+}^{\alpha} \subseteq A_{+}^{\alpha \delta}$. Hence,

$$
A_{+}^{\alpha \delta}=A_{+}^{\alpha} \oplus\left(A_{-}^{\alpha} \cap A_{+}^{\alpha \delta}\right) ;
$$

let $x \in A_{-}^{\alpha} \cap A_{+}^{\alpha \delta}$. Then $x=x \alpha \delta=-x \delta$, and this implies $2 x=x-x \delta=x(1-\delta)$ $\in A_{-}^{\alpha} \cap A_{+}^{\alpha}=0$. Since $p \neq 2$, we get $x=0$; hence, $A_{-}^{\alpha} \cap A_{+}^{\alpha \delta}=0$ and we have shown that $A_{+}^{\alpha}=A_{+}^{\alpha \delta}$.

Now assume that $\beta=-\alpha \delta$; then $A_{+}^{\alpha}=A_{+}^{\alpha \delta}=A_{-}^{-\alpha \delta}=A_{-}^{\beta}$, and this implies $A_{+}^{\alpha} \cap A_{+}^{\beta}=0$ and $\Sigma_{+}^{\alpha} \cap \Sigma_{+}^{\beta}=1$. Since $\Sigma_{+}^{\alpha}=\Sigma_{+}^{\beta}$, we get $\Sigma_{+}^{\alpha}=1$ and $\alpha=1$ or $\alpha=-1$, that is, $B=0$ or $B=A$, a contradiction.

Consequently, we have $\beta=\alpha \delta$ and $B=A_{+}^{\alpha}=A_{+}^{\alpha \delta}=A_{+}^{\beta}=C$, what we wanted to show.

Definition. Let $A$ be a reduced Abelian $p$-group with $p>3$ and let $\Gamma=$ Aut $A$. Let $H A$ be the set of all nontrivial direct summands of $A$ and let $H \Gamma$ be the set of all subgroups $\Delta \neq 1$ of $\Gamma$ such that $\Delta$ is $\alpha$-maximal and $\alpha$-indecomposable for some involution $\alpha \in \Gamma$. Then, by Theorem 3.4, $H \Gamma$ is the set of all stabilizers of elements of $H A$. Let $\Sigma_{A}: H A \rightarrow H \Gamma$ be defined by $D \rightarrow \Sigma_{A} D$ for all $D \in H A$. Then $\Sigma_{A}$ is a mapping of $H A$ onto $H \Gamma$, and by Lemma 3.5, $\Sigma_{A}$ is one-to-one. Let $S_{A}: H \Gamma \rightarrow H A$ be the inverse of $\Sigma_{A}$.

Proposition 3.6. Let $A$ and $B$ be reduced Abelian p-groups with $p>3$ and let $\gamma$ be an isomorphism of $A$ onto $B$. Then we have $\gamma^{-1}\left(\Sigma_{A} D\right) \gamma=\Sigma_{B}(D \gamma)$ for all $D \in H A$. 
In particular, if $\gamma$ is an automorphism of $A$, then we have

(a) $\Sigma_{A}(D \gamma)=\left(\Sigma_{A} D\right)^{\gamma}$ for all $D \in H A$ and

(b) $S_{A}\left(\Delta^{\gamma}\right)=\left(S_{A} \Delta\right) \gamma$ for all $\Delta \in H$ Aut $A$.

The straightforward proof is left to the reader.

4. Induced projectivities.

Definition. Let $A$ be a reduced Abelian $p$-group with $p>3$, let $B$ be a reduced Abelian $q$-group with $q>3$ and let $\phi$ be an isomorphism of Aut $A$ onto Aut $B$. Let $\Delta \in H$ Aut $A$. Then, by Theorem 3.4, there exists an involution $\alpha \in$ Aut $A$ such that $\Delta$ is $\alpha$-maximal and $\alpha$-indecomposable. Since $\phi$ is an isomorphism, $\alpha^{\phi} \in$ Aut $B$ is an involution such that $\Delta^{\phi}$ is $\alpha^{\phi}$-maximal and $\alpha^{\phi}$-indecomposable. By Theorem 3.4, we have $\Delta^{\phi} \in H$ Aut $B$. We define the mapping $\phi^{\prime}: H A \rightarrow H B$ by $D \phi^{\prime}=S_{B}\left[\left(\Sigma_{A} D\right)^{\phi}\right]$ for all $D \in H A$.

Proposition 4.1. Let $A, B$ and $C$ be reduced primary Abelian groups of characteristic greater than 3 and let $\phi:$ Aut $A \rightarrow$ Aut $B$ and $\psi:$ Aut $B \rightarrow$ Aut $C$ be isomorphisms. Then we have $(\phi \psi)^{\prime}=\phi^{\prime} \psi^{\prime}$.

Proof. For all $D \in H A$, we have

$$
\begin{aligned}
D \phi^{\prime} \psi^{\prime} & =\left(D \phi^{\prime}\right) \psi^{\prime}=S_{C}\left[\left(\Sigma_{B} D \phi^{\prime}\right)^{\psi}\right]=S_{C}\left[\left(\Sigma_{B} S_{B}\left[\left(\Sigma_{A} D\right)^{\phi}\right]\right)^{\psi}\right] \\
& =S_{C}\left[\left(\Sigma_{A} D\right)^{\phi \psi}\right]=D(\phi \psi)^{\prime} ;
\end{aligned}
$$

hence, $(\phi \psi)^{\prime}=\phi^{\prime} \psi^{\prime}$.

Proposition 4.2. Let $A$ be a reduced Abelian $p$-group with $p>3$, let $B$ be a reduced Abelian q-group with $q>3$, let $\phi$ be an isomorphism of Aut $A$ onto Aut $B$ and suppose that $A$ is not cyclic. Then we have

(a) $p=q$,

(b) $\phi^{\prime}$ is a one-to-one mapping of $H A$ onto $H B$, and $\left(\phi^{\prime}\right)^{-1}=\left(\phi^{-1}\right)^{\prime}$,

(c) $[D \gamma] \phi^{\prime}=\left[D \phi^{\prime}\right] \gamma^{\phi}$ for all $D \in H A$ and all $\gamma \in$ Aut $A$.

Proof. From Proposition 4.1 we get $D=D \phi^{\prime}\left(\phi^{-1}\right)^{\prime}$ and $E=E\left(\phi^{-1}\right)^{\prime} \phi^{\prime}$ for all $D \in H A$ and all $E \in H B$, and this proves (b).

Let $D \in H A$ and let $\gamma \in$ Aut $A$. Then $\Sigma_{A}(D \gamma)=\left(\Sigma_{A} D\right)^{\gamma}$ by Proposition 3.6(a), and this implies $\left[\Sigma_{A}(D \gamma)\right]^{\phi}=\left[\left(\Sigma_{A} D\right)^{\phi}\right]^{\gamma^{\phi}}$. Hence, $(D \gamma) \phi^{\prime}=S_{B}\left\{\left[\left(\Sigma_{A} D\right)^{\phi}\right]^{\gamma \phi}\right\}=\left(D \phi^{\prime}\right) \gamma^{\phi}$ by Proposition 3.6(b) and this is (c).

Since $A$ is not cyclic, we have $H A \neq \varnothing$; let $D \in H A$. Then $D \phi^{\prime} \in H B$ and thus $\Sigma_{A} D \simeq \Sigma_{B} D \phi^{\prime}$. Since the maximal torsion subgroup of $\Sigma_{A} D$ is a nonzero $p$-group and since the maximal torsion subgroup of $\Sigma_{B} D \phi^{\prime}$ is a nonzero q-group, it follows that $p=q$ and this is (a).

Proposition 4.3. Let $A$ and $B$ be reduced Abelian p-groups with $p>3$, let $\phi:$ Aut $A \rightarrow$ Aut $B$ be an isomorphism and let $D, E \in H A$ such that $D \subseteq E$. Then $D \phi^{\prime} \subseteq E \phi^{\prime}$ or $E \phi^{\prime} \subseteq D \phi^{\prime}$. 
Proof. We may clearly assume that $D \subset E$. Let $A=D \oplus C$; then $E=D \oplus(E \cap C)$, and there exist commuting involutions $\alpha, \beta$ of $A$ such that $D=A_{+}^{\alpha}$ and $E=A_{+}^{\beta}$. Since $A_{+}^{\alpha} \subset A_{+}^{\beta}$ and since $A_{+}^{\beta}=A_{-}^{-\beta}$, from Proposition 1.4 it follows that $A_{+}^{\alpha} \cap A_{+}^{-\beta}$ $=A_{+}^{\alpha} \cap A_{-}^{\beta}=0$, and from Proposition 1.5 we infer that

$$
\Sigma_{+}^{\alpha} \cap \Sigma_{-}^{\beta}=1 .
$$

Now $\left(\Sigma_{A} D\right)^{\phi}=\left(\Sigma_{+}^{\alpha}\right)^{\phi}$, and from Lemma 3.3 and Theorem 3.4 it follows that either

$$
D \phi^{\prime}=S_{B} \Sigma_{+}^{\alpha^{\phi}}=B_{+}^{\alpha^{\phi}}
$$

or

$$
D \phi^{\prime}=S_{B} \Sigma_{-}^{\alpha^{\phi}}=B_{-}^{\alpha^{\phi}} ;
$$

and similarly, we have either

$$
E \phi^{\prime}=B_{+}^{\beta^{\phi}}
$$

or

$$
E \phi^{\prime}=B_{-}^{\beta^{\phi}} .
$$

Hence, we have to consider four cases:

Case 12. $D \phi^{\prime}=B_{+}^{\alpha^{\phi}}$ and $E \phi^{\prime}=B_{+}^{\beta^{\phi}}$. Then $\left(\Sigma_{+}^{\alpha}\right)^{\phi}=\Sigma_{+}^{\alpha_{+}^{\phi}}$ and $\left(\Sigma_{+}^{\beta}\right)^{\phi}=\Sigma_{+}^{\beta_{+}^{\phi}}$, and from Lemma 3.3 and Theorem 3.4 it follows that $\left(\Sigma_{-}^{\alpha}\right)^{\phi}=\Sigma_{-}^{\alpha^{\phi}}$ and $\left(\Sigma_{-}^{\beta}\right)^{\phi}=\Sigma_{-}^{\beta^{\phi}}$. Now $\left(^{*}\right)$ implies $\Sigma_{+}^{\alpha^{\phi}} \cap \Sigma_{-}^{\beta^{\phi}}=1$ and since $\Sigma_{-}^{\beta^{\phi}}=\Sigma_{+}^{-\beta^{\phi}}$ and $B_{-}^{\beta^{\phi}}=B_{+}^{-\beta^{\phi}}$, we conclude that

$$
B_{+}^{\alpha^{\phi}} \cap B_{-}^{\beta^{\phi}}=0 \quad \text { or } \quad B_{-}^{\alpha^{\phi}} \cap B_{+}^{\beta^{\phi}}=0
$$

by Proposition 1.5, and from Proposition 1.4 we infer that

$$
B_{+}^{\alpha^{\phi}} \subset B_{+}^{\beta_{+}^{\phi}} \text { or } B_{+}^{\beta^{\phi}} \subset B_{+}^{\alpha^{\phi}} .
$$

Hence, $D \phi^{\prime} \subset E \phi^{\prime}$ or $E \phi^{\prime} \subset D \phi^{\prime}$, as desired.

The remaining three cases follow exactly the pattern of Case 12 and are left to the reader.

Terminological note. In the following, a subgroup $U \subseteq A$ is called cocyclic [in $A$ ] if and only if $A / U$ is cyclic.

Lemma 4.4. Let $A$ and $B$ be reduced Abelian ( $p p$ )-groups with $p>3$ and let $\phi:$ Aut $A \rightarrow$ Aut $B$ be an isomorphism. Then we have

(a) If $P \in H A$ is cyclic or cocyclic, then $P \phi^{\prime}$ is cyclic or cocyclic.

(b) $\phi^{\prime}$ preserves orders and indices of cyclic and cocyclic direct summands.

(c) $\phi^{\prime}$ is either an order-preserving or an order-reversing mapping of $H A$ onto $H B$.

Of course, (c) means that either $D \phi^{\prime} \subseteq E \phi^{\prime}$ for all $D, E \in H A$ with $D \subseteq E$, or $E \phi^{\prime} \subseteq D \phi^{\prime}$ for all $D, E \in H A$ with $D \subseteq E$.

Proof. Let $P \in H A$ be cyclic or cocyclic. Then, by Proposition 1.6, there exists an extreme involution $\alpha$ of $A$ such that $P=A_{+}^{\alpha}$, and since $\phi$ is an isomorphism, 
$\alpha^{\phi}$ is an extreme involution of $B$; and from Proposition 1.6 we infer that $B_{+}^{\alpha^{\phi}}$ and $B_{-}^{\alpha^{\phi}}$ are cyclic or cocyclic. Now $P \phi^{\prime}=B_{+}^{\alpha^{\phi}}$ or $P \phi^{\prime}=B_{-}^{\alpha^{\phi}}$, and thus (a) is proved.

Let $P \in H A$ be cyclic and let $A=P \oplus C$. Then $\Sigma_{A} P \simeq \operatorname{Hom}(C, P)$ by Hausen [6, Hilfssatz 1.4], and since $A$ is a ( $p p)$-group, there exists a cyclic direct summand $Q$ of $C$ with $|Q| \geqq|P|$. Hence, $|P|=\operatorname{Exp} \Sigma_{A} P$.

Let $C \in H A$ be cocyclic and let $A=C \oplus P$. Then $\Sigma_{A} C \simeq \operatorname{Hom}(P, C)$ by Hausen [6, Hilfssatz 1.4], and since $A$ is a (pp)-group, there exists a cyclic direct summand $Q$ of $C$ with $|Q| \geqq|P|$. Hence, $|A / C|=|P|=\operatorname{Exp} \Sigma_{A} C$.

Now $\Sigma_{A} P \simeq \Sigma_{B} P \phi^{\prime}$ for all $P \in H A$, and from the preceding argument we get (b). If rank $A=2$, then (c) is obvious; let rank $A>2$ in the following.

Let $P$ and $Q$ be cyclic direct summands of $A$ such that $|P|=|Q|$ and let $A=P$ $\oplus C=Q \oplus D$. Then $C \simeq D$ by Walker [10], and thus there exists a $\gamma \in$ Aut $A$ with $P \gamma=Q$; and from Proposition 4.2(c) we get $Q \phi^{\prime}=[P \gamma] \phi^{\prime}=\left[P \phi^{\prime}\right] \gamma^{\phi}$. Since $P \phi^{\prime}$ is cyclic or cocyclic, we get

(1) if $P \in H A$ is cyclic and $P \phi^{\prime}$ is cyclic, then $Q \phi^{\prime}$ is cyclic for every cyclic $Q \in H A$ with $|Q|=|P|$, and

$\left(1^{*}\right)$ if $P \in H A$ is cyclic and $P \phi^{\prime}$ is cocyclic, then $Q \phi^{\prime}$ is cocyclic for every cyclic $Q \in H A$ with $|Q|=|P|$.

Let $P$ and $Q$ be cyclic direct summands of $A$ such that $|P| \neq|Q|$ and let $A=P \oplus C$. Since $|Q| \neq|P|$, there exists a cyclic $R \in H C$ with $|R|=|Q|$, and since rank $A>2$, we have $P \subset P \oplus R \in H A$ and $R \subset P \oplus R \in H A$.

Assume that $P \phi^{\prime}$ is cyclic; then Proposition 4.3 implies $P \phi^{\prime} \subset(P \oplus R) \phi^{\prime}$. Suppose that $R \phi^{\prime}$ is cocyclic; then $(P \oplus R) \phi^{\prime} \subset R \phi^{\prime}$ by Proposition 4.3 , and this implies $P \phi^{\prime} \subset R \phi^{\prime}$. Hence, $P \subset R$ or $R \subset P$, a contradiction. Thus $R \phi^{\prime}$ is cyclic, and since $|R|=|Q|$, from (1) we infer that $Q \phi^{\prime}$ is cyclic. We conclude that

(2) if $P \phi^{\prime}$ is cyclic for some cyclic $P \in H A$, then $P \phi^{\prime}$ is cyclic for every cyclic $P \in H A$.

\section{Dualizing, we get}

$\left(2^{*}\right)$ if $P \phi^{\prime}$ is cocyclic for some cyclic $P \in H A$, then $P \phi^{\prime}$ is cocyclic for every cyclic $P \in H A$.

Let $X, Y \in H A$ such that $X \subset Y$ and let $A=X \oplus D=Y \oplus E$. Then $Y=X$ $\oplus(Y \cap D)$ and $A=X \oplus(Y \cap D) \oplus E$, and since $X \subset Y$, we have $Y \cap D \neq 0$. This implies that there exists a cyclic $P \in H A$ such that $P \subset Y$ and $P \nsubseteq X \nsubseteq P$.

Assume that $P \phi^{\prime}$ is cyclic; then $P \phi^{\prime} \subset Y \phi^{\prime}$ by Proposition 4.3. Suppose that $Y \phi^{\prime} \subset X \phi^{\prime}$; then $P \phi^{\prime} \subset X \phi^{\prime}$ and this implies $P \subset X$ or $X \subset P$, a contradiction. Hence $X \phi^{\prime} \subset Y \phi^{\prime}$, and from (2) we conclude that

(3) if $P \phi^{\prime}$ is cyclic for some cyclic $P \in H A$, then we have $X \phi^{\prime} \subseteq Y \phi^{\prime}$ for all $X, Y \in H A$ with $X \subseteq Y$.

Dualizing, we get

$\left(3^{*}\right)$ if $P \phi^{\prime}$ is cocyclic for some cyclic $P \in H A$, then we have $Y \phi^{\prime} \subseteq X \phi^{\prime}$ for all $X, Y \in H A$ with $X \subseteq Y$. 
Since $P \phi^{\prime}$ is either cyclic or cocyclic by (a), we conclude that $\phi^{\prime}$ is either orderpreserving or order-reversing, and this is (c).

REMARK. From Proposition 2.11 it follows that if $A \simeq A^{*}$ and if $\theta_{A}$ is the isomorphism of Proposition 2.11, then $\theta_{A}^{\prime}$ is order-reversing.

LemMA 4.5. Let $A$ and $B$ be reduced Abelian (pp)-groups with $p>3$, let $\phi$ be an isomorphism of Aut $A$ onto Aut $B$ and let $A_{1}, \ldots, A_{n} \in H A$ such that $A=\sum_{1 \leqq i}^{\circ}{ }_{n} A_{i}$. Then we have

(a) $B=\sum_{1 \leqq i \leqq n}^{\circ} A_{i} \phi^{\prime}$ if $\phi^{\prime}$ is order-preserving,

(b) $B^{*}=\sum_{1 \leqq i \leqq n}^{0}\left(A_{i} \phi^{\prime}\right)^{\times}$if $\phi^{\prime}$ is order-reversing.

Proof. Let $\beta_{1}, \ldots, \beta_{n}$ be involutions of $B$ such that $\beta_{i} \beta_{j}=\beta_{j} \beta_{i}$ for all $i$ and $j$. Then an obvious induction argument yields

$$
B=\sum_{\varepsilon_{i}= \pm}^{\circ}\left(B_{\varepsilon_{1}}^{\beta_{1}} \cap \cdots \cap B_{\varepsilon_{n}}^{\beta_{n}}\right) \text {; }
$$

-cf. Proposition 1.3. Let the involutions $\alpha_{1}, \ldots, \alpha_{n}$ of $A$ be defined by $\alpha_{i} \mid A_{i}=1_{A_{i}}$ for all $i$ and $\alpha_{i} \mid A_{j}=-1_{A_{j}}$ for $j \neq i$. Then we have $\alpha_{i} \alpha_{j}=\alpha_{j} \alpha_{i}$ for all $i, j$, and $A_{i}=A_{+}^{\alpha_{i}}$. By Lemma 3.3 and Theorem 3.4, for all $i$ we have $A_{i} \phi^{\prime}=B_{+}^{\beta_{i}}$, where $\beta_{i}=\alpha_{i}^{\phi}$ or $\beta_{i}=-\alpha_{i}^{\phi}$. Now $\alpha_{i} \alpha_{j}=\alpha_{j} \alpha_{i}$ for all $i, j$, and since $\phi$ is an isomorphism, we infer that $\beta_{i} \beta_{j}=\beta_{j} \beta_{i}$ for all $i$ and $j$.

Let $i \neq j$; then we have $A_{i} \cap A_{j}=0$ and hence, $\Sigma_{+}^{\alpha_{i}} \cap \Sigma_{+}^{\alpha_{j}}=1$. Since $\left(\Sigma_{+}^{\alpha_{i}}\right)^{\phi}=\Sigma_{+}^{\beta_{i}}$ for all $i$, we get $\Sigma_{+}^{\beta_{i}} \cap \Sigma_{+}^{\beta_{j}}=1$ and from Proposition 1.5 we infer that $B_{+}^{\beta_{i}} \cap B_{+}^{\beta_{j}}=0$ or $B_{-}^{\beta_{i}} \cap B_{-}^{\beta_{j}}=0$.

(a) Assume that $\phi^{\prime}$ is order-preserving, let $i \neq j$ and suppose that $B_{+}^{\beta_{i}} \cap B_{+}^{\beta_{j}} \neq 0$. Since $B_{+}^{\beta_{i}} \cap B_{+}^{\beta_{1}}$ is a direct summand of $B$, there exists a cyclic $P \in H A$ such that $P \subseteq B_{+}^{\beta_{i}} \cap B_{+}^{\beta_{j}}=A_{i} \phi^{\prime} \cap A_{j} \phi^{\prime}$, and this implies

$$
0 \neq P\left(\phi^{\prime}\right)^{-1} \subseteq A_{i} \cap A_{j}=0,
$$

a contradiction. Consequently, $B_{+}^{\beta_{i}} \cap B_{+}^{\beta_{j}}=0$ for all $i \neq j$, and from Proposition 1.4 we infer that $B_{+}^{\beta_{i}} \subseteq B_{-}^{\beta_{j}}$ for all $i \neq j$. Hence, $(+)$ reduces to

$$
B=\left(B_{-}^{\beta_{1}} \cap \cdots \cap B_{-}^{\beta_{n}}\right) \oplus \sum_{1 \leqq i \leqq n}^{\circ} B_{+}^{\beta_{i}}
$$

thus $\sum_{1 \leqq i \leqq n}^{\circ} A_{i} \phi^{\prime}$ is a direct summand of $B$, and this implies $B=\sum_{1 \leqq i \leqq n}^{\circ} A_{i} \phi^{\prime}$, as desired.

(b) Suppose that $\phi^{\prime}$ is order-reversing. Then we have to consider two cases:

Case 1. $n=2$. Then we have $\alpha_{2}=-\alpha_{1}$, that is, $A_{1}=A_{+}^{\alpha_{1}}$ and $A_{2}=A_{-}^{\alpha_{1}}$. Hence, $A_{2} \phi^{\prime}=B_{-}^{\beta_{1}}$ and $B=A_{1} \phi^{\prime} \oplus A_{2} \phi^{\prime}$, and from Proposition 2.6 we get $B^{*}=\left(A_{1} \phi^{\prime}\right)^{\times}$ $\oplus\left(A_{2} \phi^{\prime}\right)^{\times}$.

Case 2. $n>2$. Then we have $A_{i} \oplus A_{j} \in H A$ for all $i \neq j$; and hence, $\left(A_{i} \oplus A_{j}\right) \phi^{\prime}$ $\in H B$ is contained in $A_{i} \phi^{\prime} \cap A_{j} \phi^{\prime}$. Thus $A_{i} \phi^{\prime} \cap A_{j} \phi^{\prime}=B_{+}^{\beta_{i}} \cap B_{+}^{\beta_{j}} \neq 0$ for all $i \neq j$, and this implies $B_{-}^{\beta_{i}} \cap B_{-}^{\beta_{j}}=0$ for all $i \neq j$. From Proposition 1.4 we infer that $B_{-}^{\beta_{i}}$ $\subseteq B_{+}^{\beta_{1}}$ for all $i \neq j$ and, consequently, $(+)$ reduces to

$$
B=\left(B_{+}^{\beta_{1}} \cap \cdots \cap B_{+}^{\beta_{n}}\right) \oplus \sum_{1 \leqq 1 \leqq n}^{\circ} B_{-}^{\beta_{i}}
$$


Assume that $B_{+}^{\beta_{1}} \cap \cdots \cap B_{+}^{\beta_{n}} \neq 0$. Then there exists a cyclic $P \in H B$ with $P \subseteq A_{i} \phi^{\prime}$ $=B_{+}^{\beta_{i}}$ for $i=1, \ldots, n$. Hence $P\left(\phi^{\prime}\right)^{-1} \in H A$ is cocyclic and contains $A_{i}$ for $i=1$, $\ldots, n$, a contradiction. Thus $B_{+}^{\beta_{1}} \cap \cdots \cap B_{+}^{\beta_{n}}=0$ and, hence,

$$
0=\bigcap_{1 \leqq i \leqq n} A_{i} \phi^{\prime} \quad \text { and } \quad B=A_{i} \phi^{\prime}+\bigcap_{j \neq i} A_{j} \phi^{\prime} \quad \text { for } i=1, \ldots, n \text {; }
$$

and from Proposition 2.6 we get $B^{*}=\sum_{1 \leqq i \leqq n}^{\circ}\left(A_{i} \phi^{\prime}\right)^{\times}$. Q.E.D.

Notation. $H_{0} A$ is the [ordered] subset of $H A$ consisting of all $D \in H A$ such that $D$ or $A / D$ is finite.

LEMMA 4.6. Let $A$ and $B$ be reduced Abelian (pp)-groups and let $f$ be an orderpreserving mapping of $H_{0} A$ into $H_{0} B$ such that

(1) If $A=\sum_{1 \leqq i \leqq n}^{\circ} A_{i}$ with $A_{i} \in H_{0} A$, then $B=\sum_{1 \leqq i \leqq n}^{\circ} A_{i} f$.

(2) If $P \in H_{0} A$ is cyclic, then Pf is cyclic and $|P f|=|P|$.

(3) If $A=\{u\} \oplus C$ and if $\{u\} f=\left\{u^{\prime}\right\}$, then

(3.a) For every $x \in C[o(u)]$, there exists a unique element $x^{\prime} \in C f[o(u)]$ such that $\{u+x\} f=\left\{u^{\prime}+x^{\prime}\right\}$.

(3.b) The mapping $x \rightarrow x^{\prime}$ of $C[o(u)]$ into $C f[o(u)]$ is an isomorphism of $C[o(u)]$ onto $C f[o(u)]$.

(3.c) If $x \in C[o(u)]$ such that $\{x\} \in H_{0} A$ then $\{x\} f=\left\{x^{\prime}\right\}$.

Then, if $\{u\} \in H_{0} A$ with $o(u)=p^{k}$ and $\{u\} f=\left\{u^{\prime}\right\}$, there exists an isomorphism $\psi$ of $A\left[p^{k}\right]$ onto $B\left[p^{k}\right]$ such that $u \psi=u^{\prime}$ and $P f=P \psi$ for every cyclic $P \in H_{0} A$ with $|P| \leqq p^{k}$.

The proof is an adaptation and modification of the methods of Baer [1, Theorem II.1.3]; cf. Fuchs [4, pp. 305-308].

Proof. Let $A=\{u\} \oplus C$. Then $B=\{u\} f \oplus C f$ by (1), and from (2) we infer that $o(u)=o\left(u^{\prime}\right)$. We define $\psi$ as follows: if $x \in C\left[p^{k}\right]$, then $x \psi$ is the unique element $x^{\prime}$ which exists by (3.a) with $\{u+x\} f=\left\{u^{\prime}+x^{\prime}\right\}$; and $(n u) \psi=n u^{\prime}$ for all integers $n$. By (3.b), $\psi$ is an isomorphism of $A\left[p^{k}\right]$ onto $B\left[p^{k}\right]$.

Let $P \in H_{0} A$ be cyclic with $|P| \leqq p^{k}$ and suppose that $P \subseteq C$ or $P=\{u+x\}$ with $x \in C\left[p^{k}\right]$; then we have $P f=P \psi$ by (3.c) and (3.a).

Let $P \in H_{0} A$ be cyclic with $|P| \leqq p^{k}$ and suppose that $P \nsubseteq C$ and $P \neq\{u+x\}$ for all $x \in C\left[p^{k}\right]$. Then we have $P=\left\{p^{j} u+x\right\}$, where $k>j \geqq 1$ and $x \in C\left[p^{k}\right]$; let $|P|=p^{i}$. Since $j \geqq 1$, it follows readily that $p^{i-1} x$ has height $i-1$ in $A$; and since $p^{i} x=0$, we conclude that $\{x\}$ is a direct summand of order $p^{i}$ of $A$; cf. [4, top of p. 80]. Let $C=\{x\} \oplus D$. We consider two cases:

Case 1. $i=k$. Then $A=\{x\} \oplus\{u\} \oplus D$ and $\{x\} f=\{x \psi\}$ [by (3.c)], and we have $\{x+u\} f=\{x \psi+u \psi\}$. We apply (3) to $\{x\}$ and $\{u\} \oplus D$, and from (3.b) we get

$$
P f=\left\{x+p^{j} u\right\} f=\left\{x \psi+p^{j} u \psi\right\}=P \psi
$$

as desired. 
Case 2. $i<k$. Since $A$ is a (pp)-group, there exists a cyclic direct summand $\{y\}$ of $D$ with $o(y) \geqq p^{k}$; let $D=\{y\} \oplus E$. Using (3), we find an element $y^{\prime} \in B$ such that $\{y\} f=\left\{y^{\prime}\right\},\{y+u\} f=\left\{y^{\prime}+u \psi\right\}$ and $\{u\} f=\{u \psi\}$, and thus there exists a unique $x^{\prime}$ such that $\{y+x\} f=\left\{y^{\prime}+x^{\prime}\right\}$ and $\{x\} f=\left\{x^{\prime}\right\}$. Then $\{y+(u+x)\} f=\left\{y^{\prime}+\left(u \psi+x^{\prime}\right)\right\}$ by (3.b), and from (3.c) we get $\{u+x\} f=\left\{u \psi+x^{\prime}\right\}$. But $x^{\prime} \in C\left[p^{k}\right]$, and thus $x^{\prime}=x \psi$ by the definition of $\psi$. Now $\left\{y+\left(p^{j} u+x\right)\right\} f=\left\{y^{\prime}+\left(p^{j} u \psi+x \psi\right)\right\}$ by (3.b), and (3.c) implies $P f=\left\{p^{j} u+x\right\} f=\left\{\left(p^{j} u+x\right) \psi\right\}=P \psi$, as desired. Hence, $\psi$ is an isomorphism of $A\left[p^{k}\right]$ onto $B\left[p^{k}\right]$ such that $P f=P \psi$ for every cyclic $P \in H A$ with $|P| \leqq p^{k}$. Q.E.D.

LEMMA 4.7. Let $A$ and $B$ be reduced Abelian p-groups and let $f$ be an order-preserving mapping of $H_{0} A$ into $H_{0} B$ satisfying conditions (1), (2) of Lemma 4.6, such that for every $\{u\} \in H_{0} A$ and every $u^{\prime} \in B$ with $\left\{u^{\prime}\right\}=\{u\} f$, there exists a unique isomorphism $\psi_{u, u^{\prime}}$ of $A[o(u)]$ onto $B[o(u)]$ such that $u \psi_{u, u^{\prime}}=u^{\prime}$ and $P f=P \psi_{u, u^{\prime}}$ for every cyclic $P \in H_{0} A$ with $|P| \leqq o(u)$. Then there exists an isomorphism $\psi$ of $A$ onto $B$ such that $D f=D \psi$ for every $D \in H_{0} A$.

Proof. If $A=0$, then (1) implies $B=0$; hence, we may assume $A \neq 0$ in the following. Let $M$ be the set of all positive integers $k$ such that $A$ has cyclic direct summands of order $p^{k}$ and let $k \in M$. By hypothesis, there exists an isomorphism $\psi_{k}: A\left[p^{k}\right] \rightarrow B\left[p^{k}\right]$ such that $P f=P \psi_{k}$ for every cyclic $P \in H_{0} A$ with $|P| \leqq p^{k}$. Let $j \in M$ with $k<j$ and let $A=\{u\} \oplus\{v\} \oplus C$, where $o(u)=p^{k}$ and $o(v)=p^{j}$. Then we can find $v^{\prime} \in B$ such that $u \psi_{v, v^{\prime}}=u \psi_{k}$, and hence, $\psi_{v, v^{\prime}}=\psi_{j}$ is an extension of $\psi_{k}$.

Hence, there exist isomorphisms $\psi_{k}: A\left[p^{k}\right] \rightarrow B\left[p^{k}\right]$ for all $k \in M$, such that $\psi_{j}$ is an extension of $\psi_{k}$ whenever $k \leqq j$, and such that $P \psi_{k}=P f$ for every cyclic $P \in H_{0} A$ with $|P| \leqq p^{k}$. Let $\psi$ be the map of $A$ into $B$ which coincides with $\psi_{k}$ on $A\left[p^{k}\right]$ for all $k \in M$; clearly $\psi$ is a monomorphism.

Now if $A$ is not bounded, then it is clear that $\psi$ is epimorphic, and if $A$ is bounded of exponent $p^{k}>1$ then every $x \in B$ with $o(x)=p^{k}$ generates a direct summand, so that $\operatorname{Exp} B=p^{k}=\operatorname{Exp} A$. Hence, $\psi$ is an isomorphism of $A$ onto $B$ such that $P f=P \psi$ for every cyclic $P \in H_{0} A$; and since $D \psi=D f$ for every $D \in H_{0} A$ by (1), the proof is complete.

Terminological note. A group is a ( $p p p)$-group if and only if it is a nonzero $p$ group which has three independent elements of maximal order if it is bounded.

Corollary 4.8. Let $A$ and $B$ be reduced Abelian ( $p p)$-groups with $p \neq 2$ and let $f: H A \rightarrow H B$ be an order-preserving mapping satisfying conditions (1)-(3) of Lemma 4.6. Then there exists an isomorphism $\psi$ of $A$ onto $B$ such that $D \psi=D$ for all $D \in H A$, and if $A$ is a (ppp)-group, there exists a unique projectivity $f^{*}$ of LA onto LB inducing $f$.

Proof. From Lemma 4.6 and Corollary 1.11 we infer that $A, B$ and $f$ satisfy the requirements of Lemma 4.7. Hence, there exists an isomorphism $\psi$ of $A$ onto $B$ such that $P \psi=P f$ for every cyclic $P \in H A$; and since $D \psi \subseteq D f$ for $D \subseteq H A$, it follows that $D \psi=D f$ for all $D \in H A$. 
Let $f^{*}$ be the projectivity of $L A$ onto $L B$ induced by $\psi$, let $A$ be a (ppp)-group and let $g$ be another projectivity of $L A$ onto $L B$ inducing $f$. Then $h=f^{*} g^{-1}$ is an autoprojectivity of $L A$, and since $A$ is a (ppp)-group, there exists an automorphism $\gamma$ of $A$ such that $U h=U_{\gamma}$ for all $U \in L A$; cf. [4, pp. 305-308]. In particular, we have $P=P_{\gamma}$ for every cyclic $P \in H A$; and it is an immediate consequence of Corollary 1.11 that $U=U \gamma=U h$ for all $U \in L A$. Consequently, $g=f^{*}$, completing the proof.

TheOREM 4.9. Let $A$ and $B$ be reduced Abelian ( $p p$ )-groups with $p>3$ and let $\phi$ be an isomorphism of Aut $A$ onto Aut $B$. Then

(a) if $\phi^{\prime}$ is order-preserving, there exists an isomorphism of $A$ onto $B$ inducing $\phi^{\prime}$;

(b) if $\phi^{\prime}$ is order-reversing, there exists an isomorphism of $A$ onto $B^{*}$ inducing $\phi^{\prime} \times$; and if $A$ is a (ppp)-group, then

$\left(\mathrm{a}^{\prime}\right)$ if $\phi^{\prime}$ is order-preserving, there exists a unique projectivity of $L A$ onto $L B$ which extends $\phi^{\prime}$;

$\left(b^{\prime}\right)$ if $\phi^{\prime}$ is order-reversing, there exists a unique projectivity of $L A$ onto $L B^{*}$ which extends $\phi^{\prime} \times$.

Proof. Case A. $\phi^{\prime}$ is order-preserving. Then from Lemma 4.4 and Lemma 4.5(a) we infer that $A, B$ and $\phi^{\prime}$ satisfy the requirements of Lemma 4.6, except for (3).

Let $A=\{u\} \oplus C$, let $o(u)=p^{k}$, let $\{u\} \phi^{\prime}=\left\{u^{\prime}\right\}$ and let $C \phi^{\prime}=C^{\prime}$. Let $\Delta_{A} C$ be the group of all endomorphisms $\sigma$ of $A$ such that $A \sigma \subseteq C$ and $C \sigma=0$. Then the mapping $\phi_{u, C}$ of $C\left[p^{k}\right]$ into $\Delta_{A} C$, defined by $u\left(x \phi_{u, C}\right)=x$ and $C\left(x \phi_{u, C}\right)=0$ for all $x \in C\left[p^{k}\right]$, is an isomorphism of $C\left[p^{k}\right]$ onto $\Delta_{A} C$; and in the same fashion we get an isomorphism $\phi_{u^{\prime}, C^{\prime}}$ of $C^{\prime}\left[p^{k}\right]$ onto $\Delta_{B} C^{\prime}$.

Let $\psi_{C}: \Sigma_{A} C \rightarrow \Delta_{A} C$ be defined by $\gamma \psi_{C}=\gamma-1$ for all $\gamma \in \Sigma_{A} C$; from Hausen [6, Hilfssatz 1.4] it follows that $\psi_{C}$ is an isomorphism of $\Sigma_{A} C$ onto $\Delta_{A} C$, and in the same fashion, $\psi_{C^{\prime}}$ is an isomorphism of $\Sigma_{B} C^{\prime}$ onto $\Delta_{B} C^{\prime}$.

Since $\Sigma_{B} C^{\prime}=\left(\Sigma_{A} C\right)^{\phi}$, it follows that the mapping $\alpha: C\left[p^{k}\right] \rightarrow C^{\prime}\left[p^{k}\right]$, defined by

$$
x \alpha=\left[x \phi_{u, C} \psi_{C}^{-1}\right]^{\phi} \psi_{C^{\prime}} \phi_{u^{\prime}, C^{\prime}}^{-1}
$$

for all $x \in C\left[p^{k}\right]$, is an isomorphism of $C\left[p^{k}\right]$ onto $C^{\prime}\left[p^{k}\right]$; let $\delta=\phi_{u, C} \psi_{c}^{-1}$ and $\delta^{\prime}=\phi_{u^{\prime}, C^{\prime}} \psi_{C^{\prime}}^{-1}$.

Let $x \in C\left[p^{k}\right]$. Then we have $u(x \delta)=u+x$, and from Proposition 4.2(c) we infer that $\{u+x\} \phi^{\prime}=[\{u\}(x \delta)] \phi^{\prime}=\left\{u^{\prime}\right\}(x \delta)^{\phi}$. But $(x \delta)^{\phi}=(x \alpha) \delta^{\prime}$ and, hence, $\{u+x\} \phi^{\prime}$ $=\left\{u^{\prime}\right\}(x \alpha) \delta^{\prime}=\left\{u^{\prime}+x \alpha\right\}$. Let $x^{\prime}=x \alpha$; then $x^{\prime} \in C^{\prime}\left[p^{k}\right]$ and we have $\{u+x\} \phi^{\prime}=\left\{u^{\prime}+x^{\prime}\right\}$ for all $x \in C\left[p^{k}\right]$.

Let $x^{\prime}, x^{\prime \prime} \in C^{\prime}\left[p^{k}\right]$ such that $\left\{u^{\prime}+x^{\prime}\right\}=\left\{u^{\prime}+x^{\prime \prime}\right\}$, that is, $r\left(u^{\prime}+x^{\prime}\right)=u^{\prime}+x^{\prime \prime}$ for some integer $r$. It follows that $(r-1) u^{\prime}=x^{\prime \prime}-r x^{\prime}=0$ and thus we have $r \equiv 1 \bmod p^{k}$. Hence, $r x^{\prime}=x^{\prime}$ and $x^{\prime}=x^{\prime \prime}$; and we have shown that the mapping $x \rightarrow x^{\prime}=x \alpha$ satisfies the requirements (3.a) and (3.b) of Lemma 4.6.

Let $x \in C\left[p^{k}\right]$ such that $\{x\} \in H A$. Since $x \delta \in \Sigma_{A}\{x\}$, we have $(x \delta)^{\phi} \in \Sigma_{B}\{x\} \phi^{\prime}$; hence, $x^{\prime}=u^{\prime}\left((x \delta)^{\phi}-1\right) \in\{x\} \phi^{\prime}$ and since $o(x)=o\left(x^{\prime}\right)$, we get $\{x\} \phi^{\prime}=\left\{x^{\prime}\right\}$. 
Hence, $A, B$ and $\phi^{\prime}$ satisfy all requirements of Corollary 4.8. We conclude that (a) there exists an isomorphism of $A$ onto $B$ inducing $\phi^{\prime}$ and, if $A$ is a (ppp)-group, $\left(a^{\prime}\right)$ there exists a unique projectivity of $L A$ onto $L B$ which extends $\phi^{\prime}$.

Case B. $\phi^{\prime}$ is order-reversing. Then $\phi^{\prime} \times$ is an order-preserving mapping of $H A$ into $H B^{*}$, and from Lemma 4.4 and Lemma $4.5(\mathrm{~b})$ we infer that $A, B^{*}$ and $\phi^{\prime} \times$ satisfy the requirements of Lemma 4.6, except for (3).

Let $A=\{u\} \oplus C$, let $o(u)=p^{k}$, let $C \phi^{\prime}=C^{\prime}$ and let $u^{\prime} \in B^{*}$ such that $\left[\{u\} \phi^{\prime}\right]^{\times}=\left\{u^{\prime}\right\}$. Let the isomorphism $\phi_{u, C}: C\left[p^{k}\right] \rightarrow \Delta_{A} C$ be defined as in Case $\mathrm{A}$, and define $\chi_{C}: \Delta_{A} C \rightarrow \Sigma_{A} C$ by $\sigma \chi_{C}=1-\sigma$ for all $\sigma \in \Delta_{A} C$; that $\chi_{C}$ is an isomorphism follows from Hausen [6, Hilfssatz 1.4]. Let the isomorphism $\psi_{C^{\prime}}: \Sigma_{B} C^{\prime} \rightarrow \Delta_{B} C^{\prime}$ be as in Case $\mathrm{A}$, and define $c_{u^{\prime}}: \Delta_{B} C^{\prime} \rightarrow\left[C^{\prime}\right]^{\times}\left[p^{k}\right]$ by $\sigma c_{u^{\prime}}=\sigma u^{\prime}$ for all $\sigma \in \Delta_{B} C^{\prime}$. Since $C^{\prime}$ is cyclic of order $p^{k}$, it is clear that $c_{u^{\prime}}$ is an isomorphism.

Consequently, the mapping $\beta: C\left[p^{k}\right] \rightarrow\left[C^{\prime}\right]^{\times}\left[p^{k}\right]$, defined by

$$
x \beta=\left[x \phi_{u, c} \chi_{C}\right]^{\phi} \psi_{C^{\prime}} c_{u^{\prime}}
$$

for all $x \in C\left[p^{k}\right]$, is an isomorphism of $C\left[p^{k}\right]$ onto $\left[C^{\prime}\right]^{\times}\left[p^{k}\right]$.

Let $x \in C\left[p^{k}\right]$. Then $u+x=u\left(1+x \phi_{u, C}\right)$, and consequently, we have $\{u+x\} \phi^{\prime}$ $=\{u\} \phi^{\prime}\left(1+x \phi_{u, C}\right)^{\phi}$. Now $u^{\prime}+x \beta=\left(1-x \phi_{u, C}\right)^{\phi} u^{\prime}$, and consequently,

$$
\left(1+x \phi_{u, C}\right)^{\phi}\left(u^{\prime}+x \beta\right)=u^{\prime} .
$$

We conclude that $\{u+x\} \phi^{\prime}\left(u^{\prime}+x \beta\right)=\left[\{u\} \phi^{\prime}\right] u^{\prime}=0$, that is, $u^{\prime}+x \beta \in\left[\{u+x\} \phi^{\prime}\right]^{\times}$; and since $o(u+x)=o\left(u^{\prime}+x \beta\right)$, we infer that $\left[\{u+x\} \phi^{\prime}\right]^{\times}=\left\{u^{\prime}+x \beta\right\}$. Let $x^{\prime}=x \beta$; then $x^{\prime} \in\left[C^{\prime}\right]^{\times}\left[p^{k}\right]$ and we have $\left[\{u+x\} \phi^{\prime}\right]^{\times}=\left\{u^{\prime}+x^{\prime}\right\}$ for all $x \in C\left[p^{k}\right]$.

Exactly as in Case A, it follows that the elements $x^{\prime}$ are unique; hence, the mapping $x \rightarrow x^{\prime}=x \beta$ satisfies the requirements (3.a) and (3.b) of Lemma 4.6.

Let $x \in C\left[p^{k}\right]$ such that $\{x\} \in H A$. Then, since $1-x \phi_{u, C} \in \Sigma_{A}\{x\}$, we have $\left(1-x \phi_{u, C}\right)^{\phi} \in \Sigma_{B}\{x\} \phi^{\prime} ;$ and consequently, $\left[\{x\} \phi^{\prime}\right] x^{\prime}=0$ and $x^{\prime} \in\left[\{x\} \phi^{\prime}\right]^{\times}$. But $o(x)$ $=o\left(x^{\prime}\right)$ and, hence, $\left[\{x\} \phi^{\prime}\right]^{\times}=\left\{x^{\prime}\right\}$.

Consequently, $A, B^{*}$ and $\phi^{\prime} \times$ satisfy all requirements of Corollary 4.8 . We conclude that (b) there exists an isomorphism of $A$ onto $B^{*}$ inducing $\phi^{\prime} \times$ and, if $A$ is a (ppp)-group, $\left(\mathrm{b}^{\prime}\right)$ there exists a unique projectivity of $L A$ onto $L B^{*}$ which extends $\phi^{\prime} \times$. Q.E.D.

Corollary 4.10. Let $A$ and $B$ be reduced Abelian ( $p p)$-groups with $p>3$ and let $\phi$ be an isomorphism of Aut $A$ onto Aut $B$ such that $\phi^{\prime}$ is order-reversing. Then we have $A \simeq A^{*} \simeq B^{*} \simeq B$; and if $A$ is a finite (ppp)-group, then there exists one and only one duality of $L A$ onto $L B$ which extends $\phi^{\prime}$.

Proof. By Theorem 4.9, applied to $\phi$ and $\phi^{-1}$, we have $A \simeq B^{*}$ and $B \simeq A^{*}$. Thus $A^{*} \simeq B^{* *}$ and $B^{*} \simeq A^{* *}$ and, consequently, $A \simeq A^{* *}$ and $B \simeq B^{* *} ;$ and from Lemma 2.2 we infer that $A \simeq A^{*}$ and $B \simeq B^{*}$, proving the first part.

Suppose that $A$ is a finite (ppp)-group. Then, by Theorem 4.9 , there exists a unique projectivity $f$ of $L A$ onto $L B^{*}$ extending $\phi^{\prime} \times$. Then $h=f \times$ is a duality of 
$L A$ onto $L B$ extending $\phi^{\prime} \times \times=\phi^{\prime}$ [by Corollary 2.5]; the unicity of $h$ is an obvious consequence of Theorem 4.9( $\left.\mathrm{b}^{\prime}\right)$ and Corollary 2.5 .

\section{The automorphisms of Aut $A$.}

Proposition 5.1. Let $A$ be a reduced Abelian ( $p p$ )-group with $p>3$, let $\Gamma=$ Aut $A$ and let $\phi \in$ Aut $\Gamma$. Then the following are equivalent:

(a) $D \phi^{\prime}=D$ for all $D \in H A$,

(b) $\phi$ induces the identity automorphism in $\Gamma / c \Gamma$.

Proof. Suppose that $\phi$ satisfies (a). Then we have $D \gamma=[D \gamma] \phi^{\prime}=\left[D \phi^{\prime}\right] \gamma^{\phi}=D \gamma^{\phi}$ for all $\gamma \in \Gamma$ and all $D \in H A$ by Proposition 4.2(c). Hence, $D=D \gamma^{\phi} \gamma^{-1}$ for all $\gamma \in \Gamma$ and all $D \in H A$. From Corollary 1.11 we infer that $\gamma^{\phi} \gamma^{-1} \in c \Gamma$ for all $\gamma \in \Gamma$, and this is (b).

Suppose that $\phi$ satisfies (b). Then, if $\alpha \in \Gamma$ is an involution, we have $\alpha^{\phi}=\alpha$ or $\alpha^{\phi}=-\alpha$ since $\alpha^{\phi}$ is an involution and $\alpha^{\phi} \alpha \in c \Gamma$. Let $1 \neq \Sigma \subseteq \Gamma$ be the stabilizer of some direct summand of $A$ and let $\alpha \in \Gamma$ be an involution such that $\Sigma=\Sigma_{+}^{\alpha}$; then the preceding argument shows that $\Sigma^{\phi}=\Sigma_{+}^{\alpha}$ or $\Sigma^{\phi}=\Sigma_{-}^{\alpha}$.

Now suppose that $\Sigma^{\phi}=\left(\Sigma_{+}^{\alpha}\right)^{\phi}=\Sigma_{-}^{\alpha}$ and let $1 \neq 1+\sigma \in \Sigma_{+}^{\alpha}$. Then $A \sigma \subseteq A_{+}^{\alpha}$ and $A_{+}^{\alpha} \sigma=0$, and we have $(1+\sigma)^{\phi}=\mu(1+\sigma) \in \Sigma_{-}^{\alpha}$, where $\mu \in c \Gamma$. Hence,

$$
A_{+}^{\alpha}(\mu-1+\mu \sigma) \subseteq A_{-}^{\alpha} \text { and } A_{-}^{\alpha}(\mu-1+\mu \sigma)=0 .
$$

Now we have $A_{+}^{\alpha} \mu \sigma=0$ and $A_{-}^{\alpha} \mu \sigma \subseteq A_{+}^{\alpha}$, and this implies $A_{+}^{\alpha}(\mu-1)=0$ and $A_{-}^{\alpha}(\mu-1)$ $=0$. Hence, $\mu=1$ and $1 \neq 1+\sigma \in \Sigma_{+}^{\alpha} \cap \Sigma_{-}^{\alpha}=1$, a contradiction. This implies $\Sigma^{\phi}=\Sigma$ for all $\Sigma \in H \Gamma$, that is, $D \phi^{\prime}=D$ for all $D \in H A$, completing the proof.

Definition. Let $A$ and $B$ be groups and let $\phi:$ Aut $A \rightarrow$ Aut $B$ be an isomorphism. Then $\phi$ is an induced isomorphism if, and only if, there exists an isomorphism $\eta: A \rightarrow B$ inducing $\phi$; that is, $\gamma^{\phi}=\eta^{-1} \gamma \eta$ for all $\gamma \in$ Aut $A$.

Proposition 5.2. Let $A$ and $B$ be reduced Abelian (pp)-groups with $p>3$ and let $\phi$ be an isomorphism of Aut $A$ onto Aut $B$ such that $\phi^{\prime}$ is order-preserving. Then there exists one and only one induced isomorphism $\psi$ of Aut $A$ onto Aut $B$ such that $\phi^{\prime}=\psi^{\prime}$.

The proof is an immediate consequence of Theorem 4.9, Proposition 3.6 and Corollary 1.11 .

TheOREM 5.3. Let $A$ be a reduced Abelian ( $p p$ )-group with $p>3$, let $\Gamma=$ Aut $A$, let $\Pi=$ Aut $\Gamma$ and let $\Delta$ be the normal subgroup of $\Pi$ consisting of all automorphisms of $\Gamma$ which induce the identity in $\Gamma / c \Gamma$. Then $\Pi$ splits over $\Delta$.

Proof. Let $K$ be the subset of $\Pi$ consisting of all inner automorphisms of $\Gamma$ and all automorphisms $\theta_{A} \lambda$, where $\lambda$ : Aut $A^{*} \rightarrow \Gamma$ is an induced isomorphism; since $\theta_{A} \theta_{A}^{-1}=1_{A}$, we have $\theta_{A} \lambda=\left(\theta_{A} \lambda \theta_{A}\right) \theta_{A}^{-1}$.

Let $\lambda$ : Aut $A^{*} \rightarrow \Gamma$ be an induced isomorphism and let $\eta: A^{*} \rightarrow A$ be an isomorphism inducing $\lambda$. Let $\gamma \in \Gamma$; then $\gamma^{\theta_{A} \theta_{A}}=\eta^{*} \gamma^{* *}\left(\eta^{*}\right)^{-1}$, and since $\gamma^{* *}=\nu_{A}^{-1} \gamma \nu_{A}$ by Proposition 2.3, we get

$$
\gamma^{\theta} \hat{A}^{\lambda \theta_{A}}=\left(\nu_{A}\left(\eta^{*}\right)^{-1}\right)^{-1} \gamma\left(\nu_{A}\left(\eta^{*}\right)^{-1}\right)
$$


Consequently, $\theta_{A} \lambda \theta_{A}$ is an induced isomorphism of $\Gamma$ onto Aut $A^{*}$, induced by $\nu_{A}\left(\eta^{*}\right)^{-1}$.

Let $\mu: \Gamma \rightarrow$ Aut $A^{*}$ be an induced isomorphism and let $\sigma: A \rightarrow A^{*}$ be an isomorphism inducing $\mu$.

Let $\gamma \in$ Aut $A^{*}$. Then it follows from Propositions 2.3 and 2.9 that $\gamma^{\theta_{A}^{-1}}=$

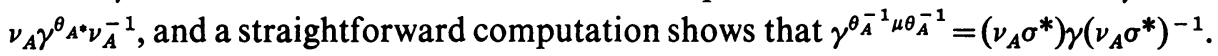
Hence, $\theta_{A}^{-1} \mu \theta_{A}^{-1}$ is an induced isomorphism of Aut $A^{*}$ onto $\Gamma$, induced by $\left(\nu_{A} \sigma^{*}\right)^{-1}$.

Now if $\lambda$ : Aut $A^{*} \rightarrow \Gamma$ is an induced isomorphism, then $\theta_{A} \lambda=\left(\theta_{A} \lambda \theta_{A}\right) \theta_{A}^{-1}$, and if $\mu: \Gamma \rightarrow$ Aut $A^{*}$ is an induced isomorphism, then $\mu \theta_{A}^{-1}=\theta_{A}\left(\theta_{A}^{-1} \mu \theta_{A}^{-1}\right)$; and since the inverses of induced isomorphisms and products of induced isomorphisms are again induced isomorphisms, it follows readily that $K$ is a subgroup of $\Pi$.

Let $\phi \in \Pi$. Then $\phi^{v} \in \Pi$ is defined as follows :

Case 1. $\phi^{\prime}$ is order-preserving. Then $\phi^{v}$ is the [by Proposition 5.2] unique inner automorphism of $\Gamma$ such that $\left(\phi^{\nu}\right)^{\prime}=\phi^{\prime}$.

Case 2. $\phi^{\prime}$ is order-reversing. Then $A \simeq A^{*}$ by Corollary 4.10 , and we have $\theta_{A}^{\prime}=\times$ by Proposition 2.11 ; thus $\phi \theta_{A}$ is an isomorphism of $\Gamma$ onto Aut $A^{*}$ such that $\left(\phi \theta_{A}\right)^{\prime}=\phi^{\prime} \times$ is order-preserving. By Proposition 5.2, there exists a unique induced isomorphism $\phi^{\lambda}$ of $\Gamma$ onto Aut $A^{*}$ such that $\left(\phi^{\lambda}\right)^{\prime}=\left(\phi \theta_{A}\right)^{\prime}$; let $\phi^{\nu}=\phi^{\lambda} \theta_{A}^{-1}$.

Now four straightforward computations, based on Proposition 4.1 and on the definition of $\lambda$, will show that $\nu$ is an endomorphism of $\Pi$. Now $\nu^{2}=\nu$ and from Proposition 5.1 we infer that $\Delta=\operatorname{ker} \nu$. Hence, $\Pi=\Delta K$ and $\Delta \cap K=1$, as desired.

REMARK. If $A$ is a (ppp)-group such that $A$ is finite if $A \simeq A^{*}$, then the subgroup $K$ of $\Pi$ constructed above is isomorphic to the group of all auto-projectivities and auto-dualities of $L A$.

\section{REFERENCES}

1. R. Baer, A unified theory of projective spaces and finite abelian groups, Trans. Amer. Math. Soc. 52 (1942), 283-343. MR 4, 109.

2. —, Linear algebra and projective geometry, Academic Press, New York, 1952. MR 14, 675 .

3. J. Dieudonné, La géométrie des groupes classiques, 2nd rev. ed., Springer-Verlag, Berlin, 1963. MR 28 \#1239.

4. L. Fuchs, Abelian groups, Akad. Kiado, Budapest, 1958; republished by Internat. Series of Monographs on Pure and Appl. Math., vol. 34, Pergamon Press, New York, 1960. MR 21 \#5672; MR 22 \#2644.

5. —, Infinite abelian groups. Vol. I, Pure and Appl. Math., vol. 36, Academic Press, New York, 1970. MR 41 \#333.

6. J. Hausen, Automorphismengesättigte Klassen abzählbarer abelschen Gruppen, Sympos. Studies on Abelian Groups (Montpellier, 1967), Springer, Berlin, 1968, pp. 147-181. MR 39 \#5691.

7. T. Koyama and J. Irwin, On topological methods in abelian groups, Sympos. Studies on Abelian Groups (Montpellier, 1967), Springer, Berlin, 1968, pp. 207-222. MR 39 \#5692.

8. H. Leptin, Abelsche p-Gruppen und ihre Automorphismengruppen, Math. Z. 73 (1960), 235-253. MR 22 \#730. 
9. R. S. Pierce, Homomorphisms of primary abelian groups, Proc. Sympos. Topics in Abelian Groups (New Mexico State Univ., 1962), Scott, Foresman, Chicago, Ill., 1963, pp. 215-310. MR 31 \#1299.

10. E. A. Walker, Cancellation in direct sums of groups, Proc. Amer. Math. Soc. 7 (1956), 898-902. MR 18, 403.

Department of Mathematics, University of TeXas at Austin, Austin, Texas 78712

Current address: Universität Trier-Kaiserslautern, Fachbereich Mathematik, 675 Kaiserslautern, Pfaffenbergstrasse 95, Federal Republic of Germany 